\title{
Clustering of patient comorbidities within electronic medical records enables high-precision COVID-19 mortality prediction
}

Aldo Faisal ( $\nabla$ a.faisal@imperial.ac.uk)

Imperial College London https://orcid.org/0000-0003-0813-7207

Erwann Le Lannou

Imperial College London https://orcid.org/0000-0003-2236-1862

Benjamin Post

Imperial College London https://orcid.org/0000-0003-0337-0827

Shlomi Haar

Imperial College London https://orcid.org/0000-0003-2213-6585

Stephen Brett

Imperial College London https://orcid.org/0000-0003-4545-8413

Balasundaram Kadirvelu

Imperial College London

Article

Keywords: COVID-19, mortality prediction, comorbidities

Posted Date: April 6th, 2021

DOI: https://doi.org/10.21203/rs.3.rs-374482/v1

License: (c) (1) This work is licensed under a Creative Commons Attribution 4.0 International License.

Read Full License 
Clustering of patient comorbidities within electronic medical records enables high-precision COVID19 mortality prediction

Erwann Le Lannou [1], Benjamin Post MD [2, 3], Shlomi Haar [1, 4, 6], Stephen J. Brett MD [5], Balasundaram Kadirvelu [1,2] \& A. Aldo Faisal [1, 2, 3, 6]

1: Brain \& Behaviour Lab: Department of Bioengineering, Imperial College London, London, UK

2: Brain \& Behaviour Lab: Department of Computing, Imperial College London, London, UK

3: UKRI Centre for Doctoral Training in AI for Healthcare, Imperial College London, London, UK

4: Department of Brain Sciences, Imperial College London, London, UK

5: Department of Surgery and Cancer, Imperial College, London, UK

6: Behaviour Analytics Lab, Data Science Institute, London, UK

\title{
Acknowledgements
}

We are grateful to UK Biobank participants. This research has been conducted using the UK Biobank Resource under Application Number 21770. Infrastructure support for this research was provided by the Imperial NIHR Biomedical Research Centre. AAF acknowledges his UKRI Turing AI Fellowship (EP/V025449/1). The acknowledged parties and funders had no role in the design of the study.

\section{Author Contributions}

Conception and design: ELL, SH, AAF; data analysis: ELL, SH, BK, AAF; data interpretation: ELL, $\mathrm{BP}, \mathrm{SH}, \mathrm{SJB}, \mathrm{AAF}$; writing and revising the paper: ELL, BP, SH, SJB, BK, AAF

Conflict of Interest: The authors have declared that no competing interests exist.

Corresponding author: aldo.faisal@imperial.ac.uk (AAF)

\begin{abstract}
:
We present an explainable AI framework to predict mortality after a positive COVID-19 diagnosis based solely on data routinely collected in electronic healthcare records (EHRs) obtained prior to diagnosis. We grounded our analysis on the $1 \frac{1}{2}$ Million people UK Biobank and linked NHS COVID19 records. We developed a method to capture the complexities and large variety of clinical codes present in EHRs, and we show that these have a larger impact on risk than all other patient data but age. We use a form of clustering for natural language processing of the clinical codes, specifically,
\end{abstract}


topic modelling by Latent Dirichlet Allocation (LDA), to generate a succinct digital fingerprint of a patient's full secondary care clinical history, i.e. their comorbidities and past interventions. These digital comorbidity fingerprints offer immediately interpretable clinical descriptions that are meaningful, e.g. grouping cardiovascular disorders with common risk factors but also novel groupings that are not obvious. The comorbidity fingerprints differ in both their breadth and depth from existing observational disease associations in the COVID-19 literature. Taking this data-driven approach allows us to avoid human-induction bias and confirmation bias during selection of what are important potential predictors of COVID-19 mortality. Together with age, these digital fingerprints are the single most important factor in our predictor. This holds the potential for improving individual risk profiling for clinical decisions and the identification of groups for public health interventions such as vaccine programmes. Combining our digital precondition fingerprints with demographic characteristics allow us to match or exceed the performance of existing state-of-the-art COVID-19 mortality predictors (EHCF) which have been developed through expert consensus. Our precondition fingerprinting and entire mortality prediction analytics pipeline are designed so as to be rapidly redeployable, e.g. for COVID-19 variants or other pre-existing diseases. 


\section{Introduction}

The outbreak of the novel, severe and acute respiratory syndrome, Coronavirus 2 (SARS-CoV-2), and its associated disease COVID-19, in Wuhan China, has presented an important and urgent threat to global health since December 2019. Declared a pandemic by the World Health Organisation (WHO) in early March $^{1}$, this disease has, as of 8 March 2021, caused 2,582,528 deaths from 116,166,652 cumulative worldwide cases ${ }^{2}$. On the same day in the United Kingdom (UK) SARS-CoV-2 is reported as responsible for 124,419 deaths ${ }^{2}$. The COVID-19 outbreak has led to an increase in hospital admissions and has considerably increased the demand for both general hospital and critical care beds $^{3,4}$. This increases the need for an objective, data-driven understanding of risk factors and accurate prediction of adverse outcomes based on pre-existing information. Such understanding may facilitate better individual clinical decisions, but potentially also increase the resilience of public health policy.

Emerging evidence throughout the pandemic has identified several risk factors associated with COVID-19 clinical severity ${ }^{5}$ and fatality ${ }^{6,7}$. Most commonly reported risk factors are old age ${ }^{8-10}$, male gender ${ }^{11,12}$ and pre-existing underlying medical conditions ${ }^{6}$. In particular, the Chinese Centre for Disease Control and Prevention identified cardiovascular disease, hypertension, diabetes, respiratory disease, and numerous malignancies to be risk factors for COVID-19 fatality ${ }^{12}$. In the United States, the most commonly initially reported comorbidities were hypertension, obesity and diabetes ${ }^{9}$. Finally, a UK-wide study aiming to characterise the clinical features of patients with severe COVID-19 highlighted chronic cardiac disease, diabetes, and chronic pulmonary disease to be associated with a higher risk of clinical severity ${ }^{13-15}$. Better knowledge of an individual's risk can help better prioritise risk groups in the global vaccination effort as well as tailor our efforts for both prevention (e.g. targeted isolation or social distancing) and for treatment of confirmed cases such as immediate hospitalisation for people at greater risk. Many attempts have been made to provide risk prediction 
models for COVID-19. Already in April 2020, Wynants et al. reviewed 145 prediction models ${ }^{16}$. Of the 145 described models, 23 models were aimed at estimating the risk of mortality. The review further identified that most studies had been performed on inpatients and required lab-testing (lymphocyte count, C-reactive protein, creatinine) and so were not aimed at community-based identification. In addition, the review concluded that the currently proposed models were 'poorly reported, at high risk of bias, and their reported performance is probably optimistic', with sample sizes rarely over $1000^{16}$. In order to define who is at high risk, governmental organisations have put forward defining criteria that aim to classify low- and high-risk groups of the population ${ }^{17,18}$. However, at present these criteria are developed by 'expert consensus' based on available bodies of evidence and so require time and effort and might be biased by the experts' priors. While the comorbidities have been observed in affecting COVID-19 mortality ${ }^{19}$ they have not been systematically analysed for building a principled integrated COVID-19 mortality risk predictor. Preconditions, especially if they appear grouped, so called multi-morbidities affect a substantial proportion of the world's population ${ }^{20}$. Estimates of prevalence vary depending on population examined and definitions used, in the UK $>50 \%$ of people have at least two conditions by age 65 , and $>50 \%$ have $3+$ conditions by age $70^{21}$. Hence, we took a more principled approach to incorporating patient specific information by systematically including comorbidities and their co-occurrence in our data-driven analysis to avoid human-induction bias, while carefully avoiding and signposting the potential for AI bias further below $^{22}$. Our approach is enabled by the existence of large population datasets with linkage to registries such as death records, hospital admission and COVID-19 testing results and therefore represent a novel opportunity for automated clinical risk prediction model development on larger cohorts. Here, we used the UK Biobank ${ }^{23,24}$, which since the 16 March 2020 has linked over 26,000 COVID-19 laboratory test results electronically to its 500,000 strong cohort of participants. The use of such linked datasets has an established track record for the development and evaluation of clinical risk models, including those for cardiovascular disease, cancer, and mortality ${ }^{25-28}$. 
In this paper, we utilise the longitudinal medical records from the UK Biobank to predict COVID-19related death. We demonstrate that the data already routinely collected and stored in EHRs can be rapidly leveraged to address pressing questions related to the COVID-19 pandemic. Notably, we apply a Topic Modelling approach, Latent Dirichlet Allocation (LDA), on the entirety of the UK Biobank population to construct precise 'digital comorbidity fingerprints' (DCFs) from EHR data. Using the early data from the UK COVID-19 epidemic, we explored the utility of our approach by using the DCFs to train a Random Forrest Classifier to predict COVID-19-related death. We demonstrate the face validity of such a two-step approach to model development by comparing the prediction performance of our DCFs, combined with previously documented demographic data, to a model based on expert consensus led feature selection, and one based entirely on age. We further consider the relationship between our data-driven DCFs and clinician-driven features. The approach proposed here is designed to provide a rapid, intuitive and accurate forecasting of COVID-19-related death based on past medical records, which is of special importance for managing hospital resources, vaccination efforts and preventative policies.

\section{Results}

Characteristics of the study population

A total of 2,499 COVID-19 patients were included in this analysis (Figure 1; cohort description in Table 1). Overall, the median (IQR) age of patients at baseline was 70.4 (59.9 - 76.3, range: 50.4 83.7) years, and 1,284 patients (51.4\%) were male. Over the study period, COVID-19-related deaths were recorded in linked death registration data for 349 patients ( $14.0 \%$ of the study population). The hospitalised patients encompassed $79 \%$ of the 349 death events. Figure 1, shows the weekly number of positive test results during the study period, from January 30 to September 9, along with the weekly number of COVID-19 related deaths in the same period. We can observe a peak in early April 2020 (week of the 8 April, 272 people tested positive) followed by a peak in COVID-19 related deaths a 
week later (week of the 15 April 2020, 61 COVID-19 related deaths). The median age of the patients who died of a COVID-19 related death was 76.0 years (76.1 years for men and 75.4 years for women). The median follow-up time for all patients (time between diagnosis and either death or end of the study) was 113 days (10 days for COVID-19-related deaths and 123 days for patients still alive at the end of the study period). Figure 1 shows the overall probability of survival 28 days after diagnosis was over 0.88 , dropping to 0.82 ; whereas for participants over 80 years old, the survival probability was 0.69 after 28 days falling to 0.64 .

In order to better characterise the impact of pre-existing conditions on the study population, we used the categories from our Expert Hand-Crafted Features (EHCF) (see Box 1). The most common comorbidity in the population was hypertension (47.4\%) followed by Cardiovascular disease (39.0\%) and cancer $(21.1 \%)$ (Table 1$)$. The univariate and fully adjusted associations (calculated using logistic regression) between patient level characteristics (Table 1) and odds of COVID-19-related death are shown in Table 1 and Figure 2. Increasing age showed the strongest association with an increased likelihood of COVID-19-related death; with participants aged over 80 years being over 15 times (fully adjusted OR: 15.86, 95\% CI: 7.85 - 32.04) more likely to die of a COVID-19-related death compared to 50-59-year-olds. Other significant characteristics that showed an increased likelihood of COVID19-related death are male gender (fully adjusted OR: 1.54, 95\% CI: 1.18 - 2.01), and a previous diagnosis of hypertension (fully adjusted OR: $1.54(1.14$ - 2.09)). These findings are consistent with previously reported associations ${ }^{29-31}$.

\section{Digital Comorbidity fingerprinting}

Pre-existing conditions are coded as thousands of possible ICD-10 disease codes in patient records. Our methodology adapts unsupervised methods from Natural Language Processing (NLP) trained on the general population (i.e. the entire UK Biobank which includes over half a million patients) in order to cluster patients' conditions using only disease codes from a patient's past hospital visits. We use a form of Latent Dirichlet Allocation, a Bayesian clustering method that can naturally model the 
categorical nature and inherent imprecision of disease codes. This clustering distils thousands of disease codes, and billions of possible combinations into a set of DCFs. Each patient's pre-existing conditions are thus summarised by a single number (between $0-100 \%$ ) describing how strong a specific pre-existing condition fingerprint is present in their record. All pre-existing conditions and their myriad of combinations are summarised by a total of 30 DCFs.

We describe hereafter how a second interpretable machine learning algorithm is used to classify participants with suspected or confirmed COVID-19 into a high and low COVID-19-related death risk category based on their digital precondition fingerprint and their demographics.

We initially fitted our DCF Model on the 402,902 participants who belonged to the UK Biobank cohort, were still alive in January 2020 and were part of the Hospital Episode Statistics (HES) subdatabase. Latent Dirichlet Allocation was used to generate the topic distributions for all patients and ICD-10 code group. We tested multiple values of topics ( $k$ ranging from 2 to 100) and compared the resultant topic distribution with respect to the model coherence, $C_{v}{ }^{32}$. The best results were obtained for $k=30$ topics, and Dirichlet priors $\alpha=0.1$ and $\eta=0.6$. We clinically reviewed disease codes grouped in each of the generated topics, i.e. the DCFs. We observe that our comorbidity fingerprinting does not group codes simply by clinical system (e.g. respiratory disorders or diseases of the circulatory system) as can be seen in Figure 3. Rather, the method groups past clinical codes in a more functional way (all precondition fingerprints are listed in the Supplementary Box 2). For example, DCF 24 groups codes of cardiovascular disorders together (such as angina and myocardial infarction), but also includes some of the most common risk factors for these diseases, such as lipidaemia or family history of cardiac disorders. $D C F 7$ groups codes for rhythm disorders (e.g. atrial fibrillation) with pulmonary embolisms. Precondition fingerprint $D C F 22$ groups diabetic disorders with occurrences of renal failure and obesity. Other fingerprints, such as $D C F 5, D C F 8$ and $D C F 19$ are mainly defined by neoplasm and surgical procedures (e.g. acquired absence of organs). 
These results indicate that comorbidity fingerprinting is able to yield clinically relevant topics by grouping diseases that often appear simultaneously. Some of the clusters are harder to interpret than others and would need to be further investigated. The DCFs are further described in Figure 4, where we demonstrate the ability of the DCFs to differentiate between patients that died of COVID-19 and the overall UK Biobank cohort. Figure 4 further outlines the DCFs ability to encompass notions of gender, age and BMI. It is, nonetheless, clear that comorbidity fingerprinting does generate coherent and interpretable results when applied to disease codes.

\section{Prediction accuracy}

In order to evaluate our DCF approach to feature engineering, we used it as an input to a supervised classifier of COVID-19 mortality and assessed its discrimination capabilities when compared to a model built using the EHCF. The prediction accuracy for the two models under consideration are shown in Table 2 and Figure 5. We use a model built from age alone as a baseline for performance evaluation (area under the receiver operating curve (AUC-ROC): 0.709 95\% CI: 0.692 - 0.727). Both the EHCF Model, (AUC-ROC: 0.734, 95\% CI: 0.704 - 0.764) and the DCF Model, (AUC-ROC: 0.730, 95\% CI: $0.700-0.760$ ) achieved similar discrimination scores. A Wilcoxon's rank-sum test was performed to assess if our DCF Model showed better discrimination capabilities than the baseline. Although there was a small absolute difference between these two models (a difference of 0.021), the $p$-value was significant at 0.0409 .

\section{Model interpretability and impact}

Comparing the EHCF Model to our DCF Model showed overlap in the top contributing factors for predicting COVID-19-related death (Figure 6). Age is the feature that plays the most predominant role in both models. Nine of the other top ten ranked predictor variables (diabetes, renal disease, 
hypertension, respiratory diseases, sex, predisposition to infection and cardiovascular diseases) were identified in both models. Indeed, the EHCF Model highlights the importance of cardiovascular diseases, diabetes, sex, cardiac diseases, severe respiratory disease, chronic kidney disease, conditions that predispose to infection and cancer. These are mirrored in DCF 24 (cardiovascular diseases), fingerprint $D C F 22$, (severe diabetes and obesity), fingerprint $D C F 4$ (chronic pulmonary disease and tobacco usage), $D C F 18$ (chronic kidney failure and hypertension), DCF 0 (haematological disorders and immunosuppression). DCF 8 in the DCF Model reflects some preconditions of the female sex (acting as an indicator for the sex variable, see Figure 6) and some associated diseases by concentrating on female-specific cancer codes such as breast cancer and more generic ICD-10 cancer codes such as chemotherapy sessions. Chronic neurological conditions are important predictors in the EHCF Model but this was not directly identified as important in the DCF Model. Instead, the DCF Model puts emphasis on gastrointestinal cancers and disorders of the GI tract through the importance of $D C F 19$, this is a feature not covered in the EHCF Model. Thus, we obtained a different set of features in our comorbidity fingerprinting (DCF), as it encompassed significantly more comorbidities per topic, than feature sets assembled by-hand (EHCF).

\section{Discussion}

Our findings demonstrate how taking a strictly data driven approach using disease codes directly from EHRs can be rapidly leveraged for predicting outcomes and studying disease patterns. The model developed in this study required, in addition to the previously available data (prior to January 2020), only diagnosis information and cause of death, to predict who, with a positive COVID-19 test, was likely to die. Even though our model does not rely on symptoms, laboratory values or images (at the time of diagnosis or during the illness) to predict mortality, we are able to achieve comparably high discrimination results (ROC-AUC of 0.703). Other published COVID-19 prognosis models have AUC-ROC's ranging from 0.68 to $0.90^{33-35}$. The use of physiological data at the point of admission further makes these models difficult to implement in a community setting or use a specific predefined subset of comorbidities ${ }^{36}$. 
In order to evaluate the capabilities of our topic model-based approach to clinical dimensionality reduction, we compared it to current medical risk prediction approaches ${ }^{19,35}$. We opted to develop a set of expert hand-crafted features, built using the CDC and NHS COVID-19 shielding list combined with current research. Our method showed comparable discrimination capabilities, (ROC-AUC of 0.730 ) to the current expert models (ROC-AUC of 0.734). The significance of our AIbased methodology is highlighted by the ability of our model to encompass the entire past medical history of the participants and, in the context of a new and unknown disease, not be restricted by potentially biased prior belief. In this way, the automated, data-driven approach we present here is able to avoid human-induction bias in the classifier's input features and permits the detection of novel insights in a new disease in a very short amount of time and with minimal human supervision. Furthermore, by ensuring a fully automated process, the methodology we present here is easily scalable to other healthcare systems and generalisable to future events. Furthermore, the DCFs we present here are not specifically tied to COVID-19 risk prediction and can thus be immediately reused to summarise risk predictions for COVID-19 variants or other diseases.

Our study provides results regarding key findings in the underpinning structure of comorbidities and validates the usefulness of these associations with their predictability of COVID-19 mortality. This is of particular importance given the high UK prevalence (18.5 million individuals) of underlying conditions that increase the risk of severe COVID-19 ${ }^{37}$. Our DCFs offer a rich and deep set of features, that can succinctly summarise a patient's comorbidity profile using topic modelling. This approach allows for the use of a high-performance AI algorithm whilst remaining clinically interpretable and intuitive. Some obvious common causes of comorbidity clusters are well known (e.g. cardiovascular disease due to smoking or obesity ${ }^{38}$ ), advances in the science of multi-morbidities are challenging because diseases may have linked or independent causes, combine heterogeneously, are treated differently, and thus affect care pathways and medication strategies. Structuring the myriad of combinations of comorbidities, such as through our comorbidity fingerprinting, underpins any 
measures to slow the accumulation of conditions and to optimise their treatment. We have shown that AI methods can help in identifying multimorbidity clusters without human-induction bias, while at the same time ensure that findings remain clinically useful.

We further demonstrate that the DCFs generated using LDA correspond well and show significant variable importance overlap when compared with the EHCF Model and previously identified risk factors from published epidemiological studies. For example, our model supports the previous epidemiological findings that cardiovascular disorders and hypertension increase risk of COVID-19related death ${ }^{12,13,19}$. Several features identified in the model warrant further evaluation as they were not specifically seen in the initial COVID-19 epidemiological studies. DCF 19 'GI Disorders' was one of the most important features in our model, however many of the epidemiological studies do not report this as a significant risk factor ${ }^{13,31}$.

There are a few limitations to our study. Firstly, our data set while vast, may also reflect the inherent bias of the UK Biobank ${ }^{39}$, which has been discussed in detail elsewhere ${ }^{39-41}$; notably, the demographic reflect a "healthy volunteer" bias, with individuals being generally older, from more educated, less deprived socioeconomic backgrounds, and with significant under-representation of ethnic minorities compared to the UK population. Secondly, testing, treatments, and outcomes of COVID-19 have continuously improved during the study period, thus possibly having a confounding effect on the results. Moreover, due to the limited availability of testing kits for COVID-19, priority was initially offered to those considered at a higher clinical risk, thus potentially leading to an overestimation of severe outcomes in the database. However, these COVID-19 specific data bias factors only affect the mortality prediction but not the structure of the DCFs. This study further relied on retrospective secondary care EHRs and the model is therefore currently blind to conditions entirely managed in primary care, such conditions include many less severe cases of diabetes, asthma and hypertension. At the time of the study, data from primary care was not available. Future work will be needed to 
incorporate data from General Practices into the development of the DCFs. Balancing the strengths and limitations, we consider our derivation population to be relevant for the initial exploration of COVID-19 mortality in an older and more 'at risk' population using a machine leaning approach. Further research and external validation of the predictive models and DCFs in this study will be required before applying this work in a more general context, specifically to better asses the risk in a younger and fully diverse population.

In conclusion, we developed a novel machine learning based approach to predict COVID-19 mortality in a community cohort only from past EHR data. We have demonstrated the feasibility of a comorbidity clustering approach to avoid human-induction bias and succinctly summarise billions of possible comorbidity combinations in COVID-19 prognosis modelling and thus rapidly leverage vast EHR dataset for outcome prediction. We find, that age and qualitative co-morbidity information are very powerful mortality predictors, making other demographic or physiological data pale in comparison. This implies, that in rapid decision situations or decisions with limited information will benefit from our solution as this enables healthcare professionals to form a rapid picture from information that patients and their relatives are familiar with and which do not change rapidly, unlike data such as blood pressure. Thus, our model may enable early stratification of key clinical risk groups thus permitting earlier intervention and may help better prioritise risk groups in the global vaccination effort.

Crucially, however, our DCF framework can be applied to any other form of novel disease as the multi-morbidity features we discovered span a space that is disease agnostic.

\section{Methods}


We conducted a prospective open cohort study using the Hospital Episode Statistics for England (HES) database, COVID-19 test results and death data linked through the UK Biobank (see `Data Source'). The cohort study began on 30 January 2020, which was chosen as it corresponds to the day of the first laboratory-confirmed case of COVID-19 in the UK ${ }^{42}$; and ended on 9 September 2020. The cohort study examines risk among the population of patients who are positive for COVID-19. Therefore, we only included patients from the UK Biobank if they had a positive SARS-COV-2 test result or a COVID-19 diagnosis code recorded in their clinical notes (ICD-10 code U071 or U072).

Data source and ethical approval

The UK Biobank is a large prospective population cohort of 502,505 participants aged 40-70 years at baseline, prepared to travel to 1 of 22 assessment centres in England, Scotland, and Wales ${ }^{23}$. All participants were recruited between 2006 and 2010, and all consented to have their health followed ${ }^{23}$. Baseline assessments include nurse-led interviews surrounding socio-demographics, lifestyle, physiological measurements, and medical history. Health outcomes were sourced from linkages to electronic medical records. UK Biobank obtained approval from the North West Multi-Centre Research Ethics Committee (MREC), and the Community Health Index Advisory Group (CHIAG). All participants provided written informed consent prior to enrolment in the study. The UK Biobank protocol is available online ${ }^{43}$.

In the context of the COVID-19 pandemic, the UK Biobank has implemented a rapid and dynamic linkage between the laboratory results for COVID-19, stored on Public Health England's SecondGeneration Surveillance System, and the UK Biobank participants ${ }^{24}$. The previous hospital medical information of the study participants was obtained from linkage to the HES database (a data 
warehouse containing records of all admissions, emergency room attendances and outpatients appointments at NHS hospitals in England) ${ }^{44}$.

Access to anonymised data for the UK Biobank cohort was granted by the UK Biobank Access Management Team (application number 21770). Ethical approval was granted by the national research ethics committee (REC 16/NW/0274) for the overall UK Biobank cohort.

Outcome

The primary outcome measure was COVID-19-related death in patients with a COVID-19 infection. Confirmation of a COVID-19-related death COVID-19 death was defined as 1) a COVID-19-related death recorded in ONS death certificate data (International Classification of Disease, 10th edition, ICD-10 codes U071 or U072). 2) a death occurring within 28 days of a laboratory-confirmed COVID19 infection (as per UK government guidelines ${ }^{45}$ ).

Model Development

The performance of machine learning methods is heavily dependent on the selection of features ${ }^{46}$. For that reason, most of the effort in an analytic model is spent pre-processing, merging, customizing, and cleaning datasets. This task is made all the more complicated in EHRs as the different predictors may number the tens of thousands (over 11,700 are present in the UK Biobank). Traditional modelling approaches deal with this complexity by choosing a limited number of variables and creating custom features ${ }^{47}$. However, this is a largely manual and often labour-intensive task. Another common 
approach is to use unsupervised dimensionality reduction approaches, this is often far faster but may yield results that are less accurate or interpretable.

\section{Hand-Crafted features: Predictor variables}

The patient-level characteristics included in the development of the EHCF Model were selected from the existing health conditions listed on original population-level risk stratification method as exercised in $\mathrm{UK}^{17}$ or listed by the Centers for Disease Control and Prevention (CDC) as defining 'people at increased risk for severe illness ${ }^{18}$. This was further complemented with other emerging risk factors for severe outcomes of COVID-19 (such as raised blood pressure) $)^{9,12,13,19}$. The final predictor variables chosen are summarised in Box 1.

Demographic and lifestyle variables were also included, namely: age, sex, Townsend deprivation inde ${ }^{48}$, ethnicity, and smoking status. Age groups were categorised as 50-60, 60-70, 70-80 and 80+ years. Deprivation was measured using the Townsend Deprivation Index (grouped into quartiles based on the entire UK Biobank distribution, greater scores imply a greater degree of deprivation). Ethnicity was grouped as white and non-white (thus collapsing the UK Biobank categories mixed, asian, black or chinese), due to the otherwise small number of participants in each of the specific categories. Smoking status was determined from data from the initial UK Biobank assessment and grouped into participants that smoke or have smoked (current or previous at baseline) and participants that, at baseline, were never smokers.

The demographic variables considered as potential factors affecting COVID-19 risk and were determined using the answers given at UK Biobank initial assessment centre. Age was determined on 
30 January 2020. Information on all other covariate variables was determined using the ICD-10 codes in the linked HES database.

\section{Automated features: topic modelling}

Topic modelling was originally developed as a tool to model collections of discrete data with particular application in text modelling and Natural Language Processing (NLP). Topic modelling can discover abstract 'topics' within a collection of documents, where a topic consists of a collection of words that frequently occur together. Here we opted to train topic models using Latent Dirichlet Allocation (LDA), an unsupervised and interpretable method for topic modelling ${ }^{49,50}$. LDA is a Bayesian probabilistic model that works by taking as input a corpus of clinical codes and represents each document as a finite mixture of an underlying set of fixed topics, with each topic characterised by its distribution over words ${ }^{49,51}$.

Here, LDA is used to represent a patient $p$ as a mixture over the collection of $K$ "topics". Each topic $k$ defines a multinomial distribution over a finite vocabulary of ICD-10 diagnosis codes and is assumed to have been drawn from a Dirichlet, $\beta_{k} \sim \operatorname{Dirichlet}(\eta)$. Thus, each code $c_{p, n}$ has a given probability $\beta_{k}$ in each topic $k$. Given the topics, LDA then generates for each patient $p$ a distribution over topics $\theta_{p} \sim \operatorname{Dirichlet}(\alpha)$. In other words, each topic is determined as a distribution over closely related codes and each patient is represented as a distribution over multiple topics. For simplicity, we assumed symmetric priors on the patient topic distribution $\theta$ and the code topic distribution $\beta$.

For each participant of the UK Biobank, we considered a full longitudinal visit history to hospital, including all admissions, emergency room attendances and outpatient appointments. For each recorded hospital episode, we extracted a list of diagnosis codes, using the ICD-10. This generated a document 
for each unique patient in the UK Biobank, representing their full hospital visit history as a series of 'sentences' (one for each visit, so if a patient visit the hospital 10 times for asthma it is represented 10 times in their record.), wherein each 'word' was a unique ICD-10 diagnosis code. Thus, this way of accounting naturally weighs conditions that require more frequent visits to the hospital.

The LDA topic model was trained using as input the corpus of documents (i.e. sentences of ICD-10 diagnosis codes) for the entirety of the UK Biobank population that is not lost to follow up, still alive on 30 January 2020 and is part of the HES database ('Population part of the HES database', Fig. 1). This was chosen with the aim of generating more clinically robust and disease agnostic topics (i.e. the topics formed are not specific to any prior comorbidity). From this corpus, we used a grid-searching method to determine the optimal number $K$ of topics, and the prior value for $\theta$ and $\beta$ ( $\alpha$ and $\eta$ ) when optimising with respect to model coherence, $C_{v}$ in ${ }^{32}$ as this method has been shown to achieve the highest correlation with human topic ranking data ${ }^{32,52}$. This model was implemented using the gensim library in the Python programming language ${ }^{53}$.

\section{Development of a random forest model}

Two predictive classifiers were trained in this study, EHCF Model and DCF Model. The first, EHCF Model, uses as input the 'manually' determined participant characteristics or 'Hand-Crafted Features' (see Hand-Crafted features: Predictor variables). These were grouped into the aforementioned categories and each participant was set a binary value indicating whether or not their past medical records (extracted from the HES database) showed the occurrence of at least one of the conditions for each category. The DCF Model, on the other hand, uses the patients' distribution over the previously derived topics of an LDA topic model as input to a classifier. The LDA model was further complemented with the demographic and lifestyle variables (e.g. age). 
The models described above were trained to predict the same binary output; the patients that survived were assigned to class 0 and those that died to class 1.

In this study, we opted for use of a random forest (RF) classifier ${ }^{54}$, to determine COVID-19 associated mortality. RF is a high-performance ML algorithm that fits several decision tree classifiers on various sub-samples of the dataset and, subsequently, averages these tree predictors ${ }^{54}$. This allows for a reduction in over-fitting and improvement in accuracy. We implemented all RF models using the Scikit-lean library in python programming language ${ }^{55}$. The models' hyper-parameters ${ }^{56-59}$ were determined via grid search with AUC as the response as it yields a finer evaluation than commonly used error rates ${ }^{58}$.

The overview of the pipeline used for DCF Model is illustrated in Figure 7. Figure 7 describes the learning of topics from the data and shows how we use the distribution of each patient over each of these topics as input to a RF classifier.

Variable ranking

In order to better understand the predictor variables that may lead to increased risk of a COVID-19related death and their interactions, we use an impurity-based approach to rank the contribution of the different variables in the prediction made by the model. This resulting variable importance score reflects the relative impact that each variable has on the prediction issued by the model. 
We chose to use a RF algorithm for this analysis because it is a non-parametric algorithm that can recognise complex patterns and automatically capture nonlinear and interaction effects without specifying these a priori ${ }^{60}$.

Statistical analysis

Study participant numbers are depicted in the flow chart (Figure 1). For each of the 'Hand-Crafted' patient characteristics (see Hand-Crafted features: Predictor variables), a logistic regression model was fitted with the COVID-19-related death as the outcome in order to determine a univariate (notadjusted) Odds Ratio (OR). All patients' characteristics were subsequently included in a single multivariable logistic regression to determine the adjusted OR. All OR from the univariable and multivariable logistic regression models are reported with 95\% confidence intervals. For the purpose of this study, the logistic regression model was not considered for its predictive power but rather for its ability to simply and accurately describe the available data.

The HES data were considered complete with no missing data for our population. Participants with missing smoking information were assumed to be non-smokers. Missing deprivation score was imputed using the median value of the entire UK Biobank cohort. The participants with missing ethnicity were dropped from the study as no reliable way could be determined to input this field.

We subsequently developed RF based models in order to assess discrimination capability and compare variable importance. In order to avoid over-fitting, we evaluated the prediction accuracy of these models using 10-fold stratified cross-validation and calculated the area under the AUC-ROC as a measure of model discrimination. In every cross-validation fold, a training sample ( $90 \%$ of the participants) was used to derive the models, and then a hold-out sample (10\% of the participants) was 
used for performance evaluation. We report the mean AUC-ROC and the 95\% confidence intervals for all models. In all RF models, age and deprivation score are considered as continuous variables.

\section{Data Availability}

The UK Biobank cohort data that supports the findings of this study is available to researchers as approved by the Biobank Access Management Team.

\section{References}

1. WHO. WHO Director-General's opening remarks at the media briefing on COVID-19-11 March 2020. https://www.who.int/director-general/speeches/detail/who-director-general-sopening-remarks-at-the-media-briefing-on-covid-19---11-march-2020 (2020).

2. WHO. Coronavirus disease (COVID-19): Weekly Epidemiological Update. https://www.who.int/emergencies/diseases/novel-coronavirus-2019/situation-reports (2020).

3. Arabi, Y. M., Murthy, S. \& Webb, S. COVID-19: a novel coronavirus and a novel challenge for critical care. Intensive Care Med. 1-4 (2020).

4. Grasselli, G., Pesenti, A. \& Cecconi, M. Critical care utilization for the COVID-19 outbreak in Lombardy, Italy: early experience and forecast during an emergency response. Jama $\mathbf{3 2 3}$, $1545-1546$ (2020).

5. Jordan, R. E., Adab, P. \& Cheng, K. K. Covid-19: risk factors for severe disease and death. (2020).

6. Onder, G., Rezza, G. \& Brusaferro, S. Case-fatality rate and characteristics of patients dying in relation to COVID-19 in Italy. Jama $\mathbf{3 2 3}, 1775-1776$ (2020). 
7. Zhou, F. et al. Clinical course and risk factors for mortality of adult inpatients with COVID-19 in Wuhan, China: a retrospective cohort study. Lancet (2020).

8. Garg, S. Hospitalization rates and characteristics of patients hospitalized with laboratoryconfirmed coronavirus disease 2019—COVID-NET, 14 States, March 1--30, 2020. MMWR. Morb. Mortal. Wkly. Rep. 69, (2020).

9. Richardson, S. et al. Presenting characteristics, comorbidities, and outcomes among 5700 patients hospitalized with COVID-19 in the New York City area. Jama (2020).

10. Guan, W. et al. Clinical characteristics of coronavirus disease 2019 in China. N. Engl. J. Med. 382, 1708-1720 (2020).

11. Jin, J.-M. et al. Gender differences in patients with COVID-19: Focus on severity and mortality. Front. Public Heal. 8, 152 (2020).

12. Deng, G., Yin, M., Chen, X. \& Zeng, F. Clinical determinants for fatality of 44,672 patients with COVID-19. Crit. Care 24, 1-3 (2020).

13. Docherty, A. B. et al. Features of 16,749 hospitalised UK patients with COVID-19 using the ISARIC WHO Clinical Characterisation Protocol. medRxiv (2020).

14. House, N., Holborn, H. \& Wc, L. ICNARC report on COVID-19 in critical care. Publ. online 26, 24 (2020).

15. Patel, B. V et al. Natural history, trajectory, and management of mechanically ventilated COVID-19 patients in the United Kingdom. medRxiv (2020).

16. Wynants, L. et al. Prediction models for diagnosis and prognosis of covid-19 infection: systematic review and critical appraisal. $b m j$ 369, (2020).

17. Digital, N. Covid-19—high risk shielded patient list identification methodology. https://digital.nhs.uk/coronavirus/shielded-patient-list/methodology.

18. CDC. Covid-19: People at Increased Risk. https://www.cdc.gov/coronavirus/2019-ncov/need- 
extra-precautions/people-with-medical-conditions.html (2020).

19. Clift, A. K. et al. Living risk prediction algorithm (QCOVID) for risk of hospital admission and mortality from coronavirus 19 in adults: national derivation and validation cohort study. bmj 371, (2020).

20. The Academy of Medical Sciences. Multimorbidity: a priority for global health research. Https://Acmedsci.Ac.Uk/Policy/Policy-Projects/Multiple-Morbidities-As-a-Global-HealthChallenge (2015).

21. Barnett, K. et al. Epidemiology of multimorbidity and implications for health care, research, and medical education: A cross-sectional study. Lancet (2012) doi:10.1016/S01406736(12)60240-2.

22. Rich, A. S. \& Gureckis, T. M. Lessons for artificial intelligence from the study of natural stupidity. Nature Machine Intelligence (2019) doi:10.1038/s42256-019-0038-z.

23. Sudlow, C. et al. UK biobank: an open access resource for identifying the causes of a wide range of complex diseases of middle and old age. Plos med 12, e1001779 (2015).

24. Armstrong, J. et al. Dynamic linkage of COVID-19 test results between Public Health England's second generation surveillance system and UK biobank. Microb. genomics $\mathbf{6}$, e000397 (2020).

25. Alaa, A. M., Bolton, T., Angelantonio, E. Di, Rudd, J. H. F. \& van der Schaar, M. Cardiovascular disease risk prediction using automated machine learning: A prospective study of 423,604 UK Biobank participants. PLoS One (2019) doi:10.1371/journal.pone.0213653.

26. Weng, S. F., Vaz, L., Qureshi, N. \& Kai, J. Prediction of premature all-cause mortality: A prospective general population cohort study comparing machine-learning and standard epidemiological approaches. PLoS One (2019) doi:10.1371/journal.pone.0214365.

27. Hippisley-Cox, J. \& Coupland, C. Development and validation of risk prediction algorithms to estimate future risk of common cancers in men and women: Prospective cohort study. $B M J$ 
Open (2015) doi:10.1136/bmjopen-2015-007825.

28. Clift, A. K. et al. Development and validation of risk scores for all-cause mortality for a smartphone-based 'general health score' application: a prospective cohort study using the UK Biobank (Preprint). JMIR mHealth uHealth (2020) doi:10.2196/25655.

29. Jain, V. \& Yuan, J.-M. Systematic review and meta-analysis of predictive symptoms and comorbidities for severe COVID-19 infection. medRxiv (2020).

30. Chen, T. et al. Clinical characteristics of 113 deceased patients with coronavirus disease 2019: retrospective study. Bmj 368, (2020).

31. Wu, Z. \& McGoogan, J. M. Characteristics of and important lessons from the coronavirus disease 2019 (COVID-19) outbreak in China: summary of a report of 72314 cases from the Chinese Center for Disease Control and Prevention. Jama 323, 1239-1242 (2020).

32. Röder, M., Both, A. \& Hinneburg, A. Exploring the space of topic coherence measures. in Proceedings of the eighth ACM international conference on Web search and data mining 399408 (2015).

33. Carr, E. et al. Supplementing the National Early Warning Score (NEWS2) for anticipating early deterioration among patients with COVID-19 infection. medRxiv (2020).

34. Zhang, H. et al. Risk prediction for poor outcome and death in hospital in-patients with COVID-19: derivation in Wuhan, China and external validation in London, UK. (2020).

35. Knight, S. R. et al. Risk stratification of patients admitted to hospital in the United Kingdom with covid-19 using the ISARIC WHO Clinical Characterisation Protocol: development and validation of a multivariable prediction model for mortality. Br. Med. J. (2020).

36. Williams, R. D. et al. Seek COVER: Development and validation of a personalized risk calculator for COVID-19 outcomes in an international network. medRxiv (2020).

37. Walker, J. L. et al. UK prevalence of underlying conditions which increase the risk of severe 
COVID-19 disease: a point prevalence study using electronic health records. BMC Public Health 21, 484 (2021).

38. Vetrano, D. L. et al. Twelve-year clinical trajectories of multimorbidity in a population of older adults. Nat. Commun. (2020) doi:10.1038/s41467-020-16780-x.

39. Fry, A. et al. Comparison of sociodemographic and health-related characteristics of UK Biobank participants with those of the general population. Am. J. Epidemiol. 186, 1026-1034 (2017).

40. Weng, S. F., Reps, J., Kai, J., Garibaldi, J. M. \& Qureshi, N. Can machine-learning improve cardiovascular risk prediction using routine clinical data? PLoS One 12, e0174944 (2017).

41. Ganna, A. \& Ingelsson, E. 5 year mortality predictors in 498103 UK Biobank participants: a prospective population-based study. Lancet 386, 533-540 (2015).

42. Gov.UK. Coronavirus (COVID-19) cases in the UK. https://web.archive.org/web/20200502045059/https://coronavirus.data.gov.uk/ (2020).

43. Palmer, L. J. UK Biobank: bank on it. Lancet 369, 1980-1982 (2007).

44. Adamska, L. et al. Challenges of linking to routine healthcare records in UK Biobank. Trials 16, 1 (2015).

45. GOV.UK. New UK-wide methodology agreed to record COVID-19 deaths. (2020).

46. Bengio, Y., Courville, A. \& Vincent, P. Representation learning: A review and new perspectives. IEEE Trans. Pattern Anal. Mach. Intell. 35, 1798-1828 (2013).

47. Goldstein, B. A., Navar, A. M., Pencina, M. J. \& Ioannidis, J. Opportunities and challenges in developing risk prediction models with electronic health records data: a systematic review. $J$. Am. Med. Informatics Assoc. 24, 198-208 (2017).

48. Black, D. Inequalities in health: the Black report. (Penguin Books, 1982).

49. Blei, D. M., Ng, A. Y. \& Jordan, M. I. Latent dirichlet allocation. J. Mach. Learn. Res. 3, 993- 
$1022(2003)$.

50. Blei, D. M. Probabilistic topic models. Commun. ACM 55, 77-84 (2012).

51. Griffiths, T. L. \& Steyvers, M. Finding scientific topics. Proc. Natl. Acad. Sci. 101, 52285235 (2004).

52. Syed, S. \& Spruit, M. Full-text or abstract? Examining topic coherence scores using latent dirichlet allocation. in 2017 IEEE International conference on data science and advanced analytics (DSAA) 165-174 (2017).

53. Rehurek, R. \& Sojka, P. Software Framework for Topic Modelling with Large Corpora. Proc. Lr. 2010 Work. New Challenges NLP Fram. (2010).

54. Breiman, L. Random forests. Mach. Learn. 45, 5-32 (2001).

55. Pedregosa, F. et al. Scikit-learn: Machine learning in Python. J. Mach. Learn. Res. (2011).

56. Huang, B. F. F. \& Boutros, P. C. The parameter sensitivity of random forests. BMC Bioinformatics 17, 331 (2016).

57. Probst, P., Wright, M. N. \& Boulesteix, A.-L. Hyperparameters and tuning strategies for random forest. Wiley Interdiscip. Rev. Data Min. Knowl. Discov. 9, e1301 (2019).

58. Probst, P. \& Boulesteix, A.-L. To tune or not to tune the number of trees in random forest. $J$. Mach. Learn. Res. 18, 6673-6690 (2017).

59. Boulesteix, A.-L., Janitza, S., Kruppa, J. \& König, I. R. Overview of random forest methodology and practical guidance with emphasis on computational biology and bioinformatics. Wiley Interdiscip. Rev. Data Min. Knowl. Discov. 2, 493-507 (2012).

60. Wright, M. N., Ziegler, A. \& König, I. R. Do little interactions get lost in dark random forests? BMC Bioinformatics 17, 145 (2016). 


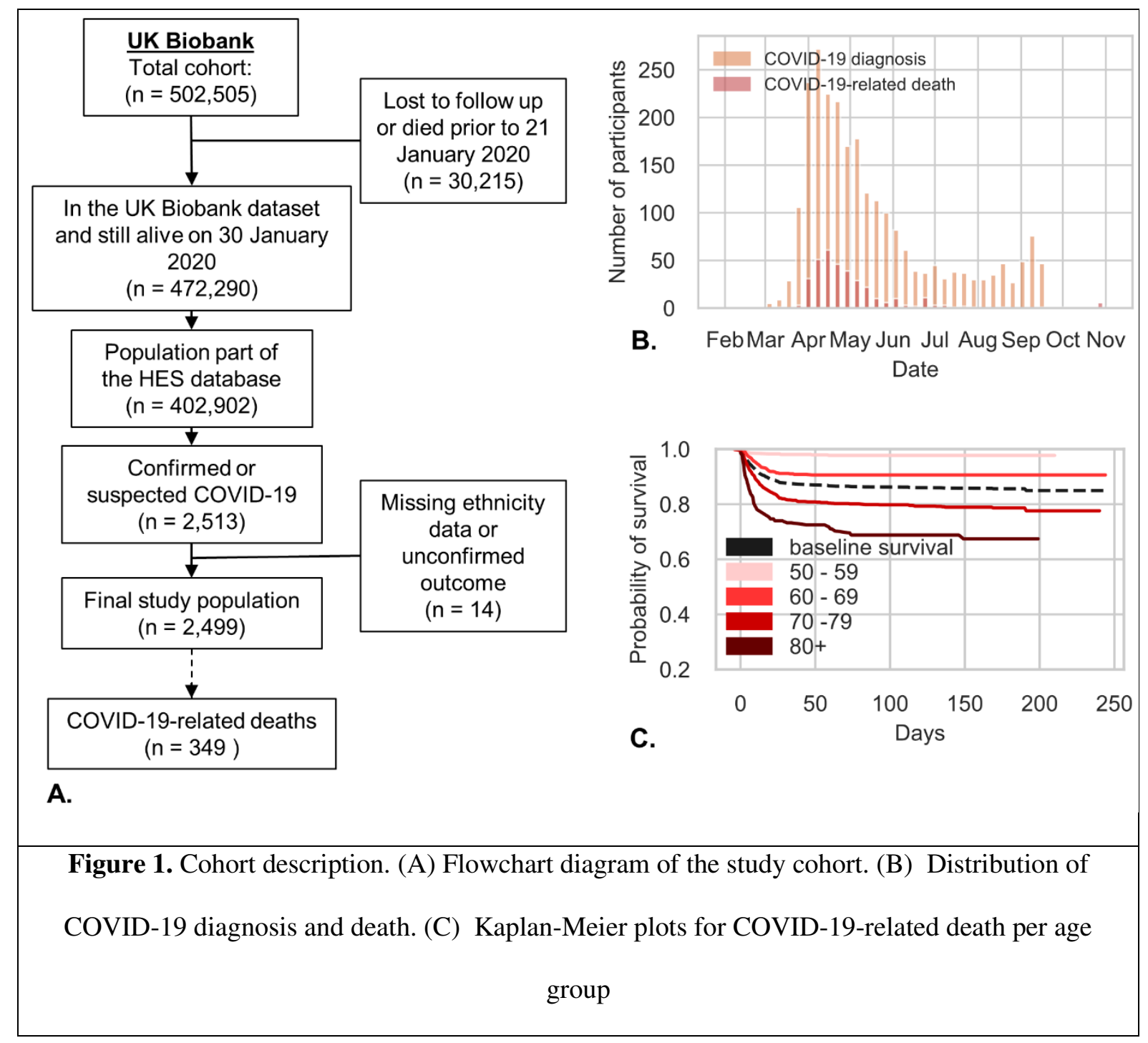




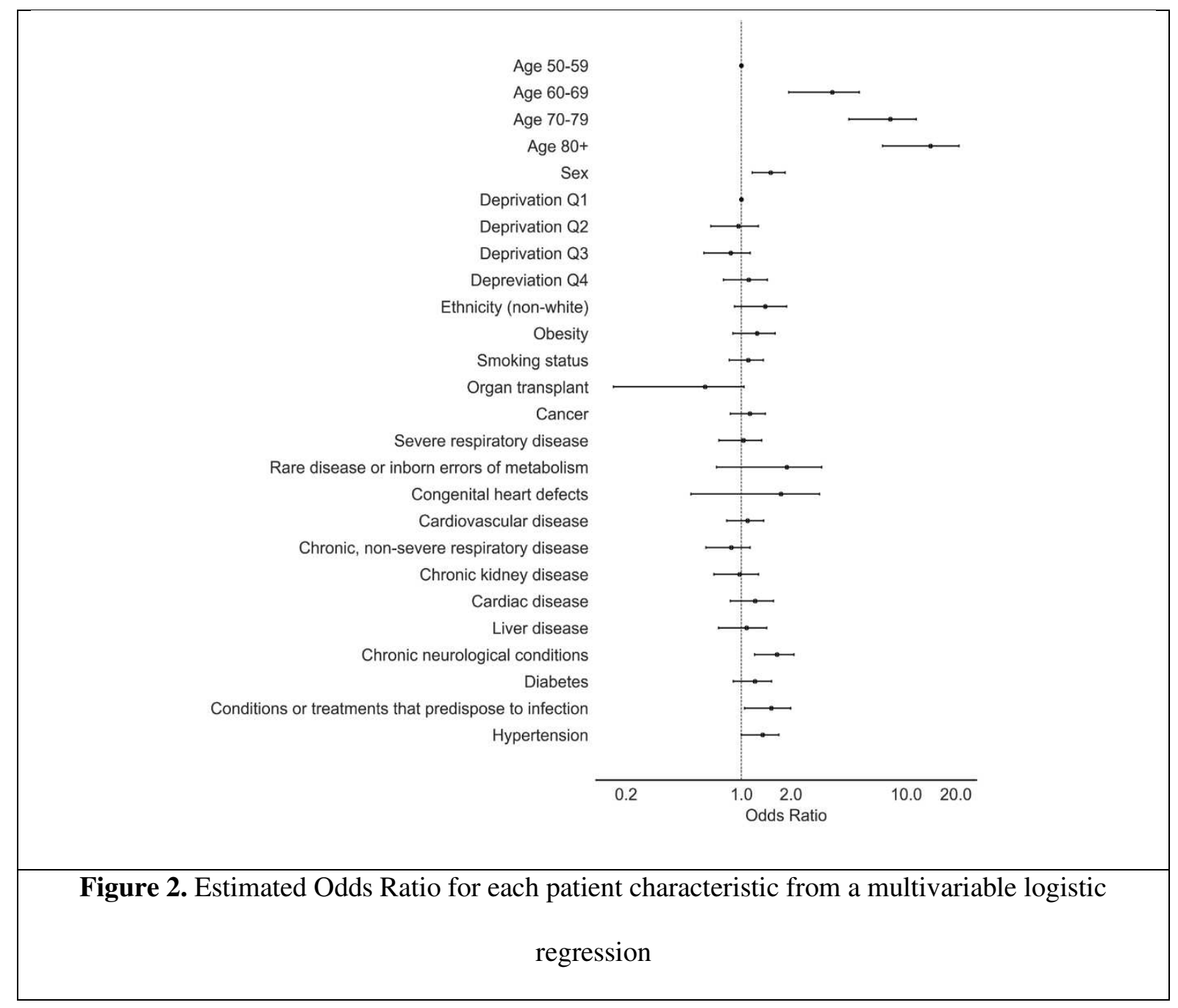




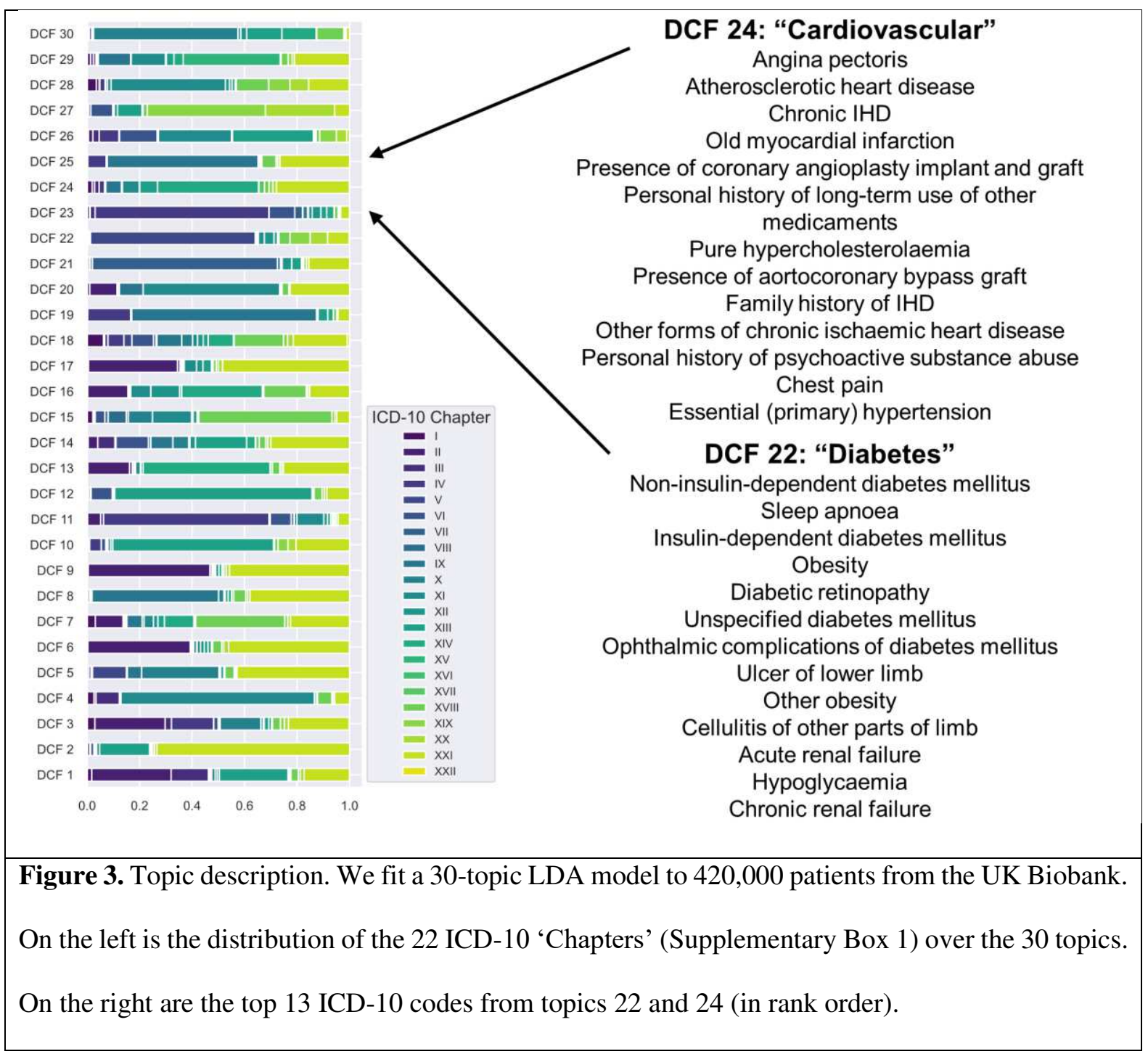




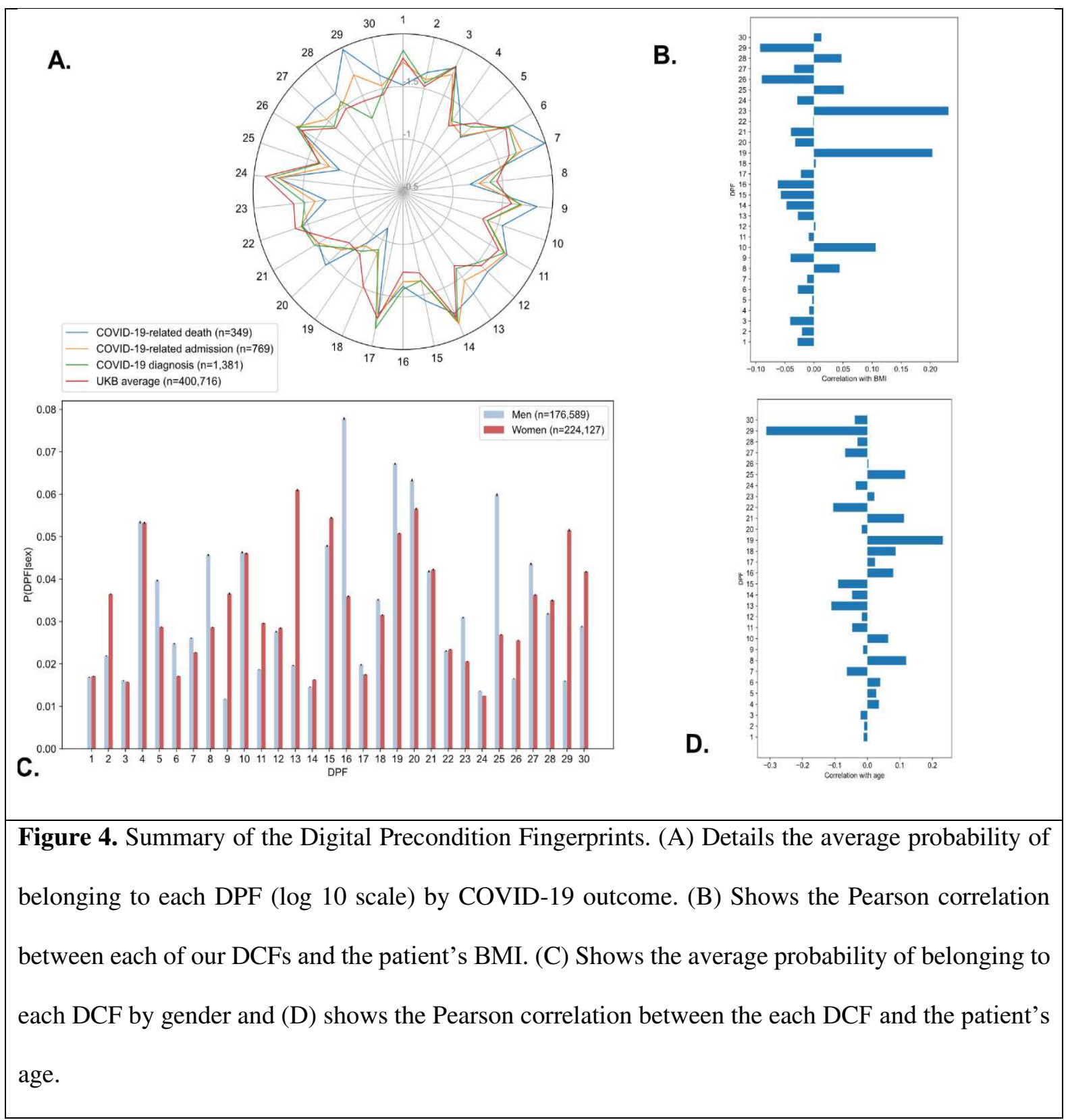




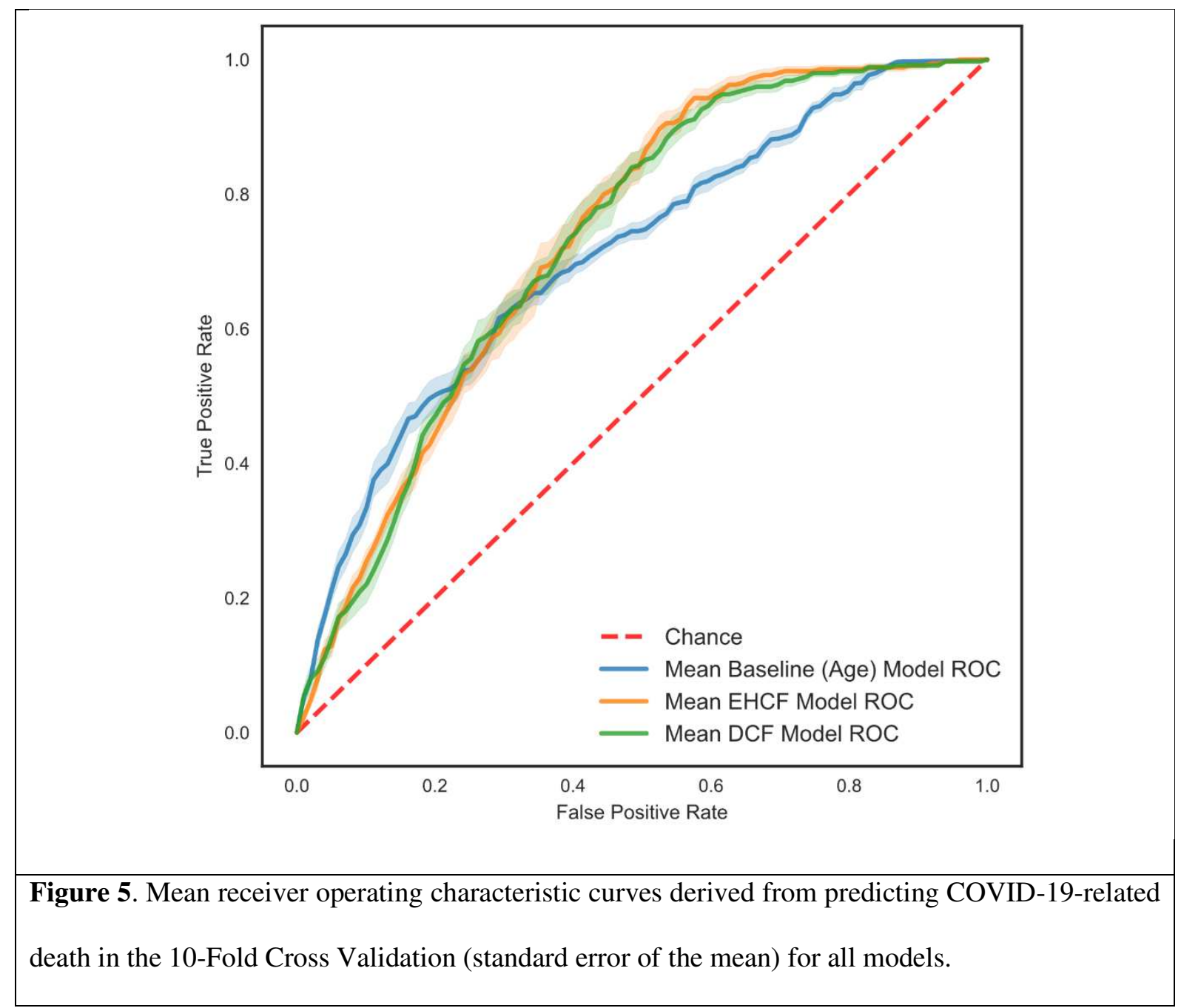




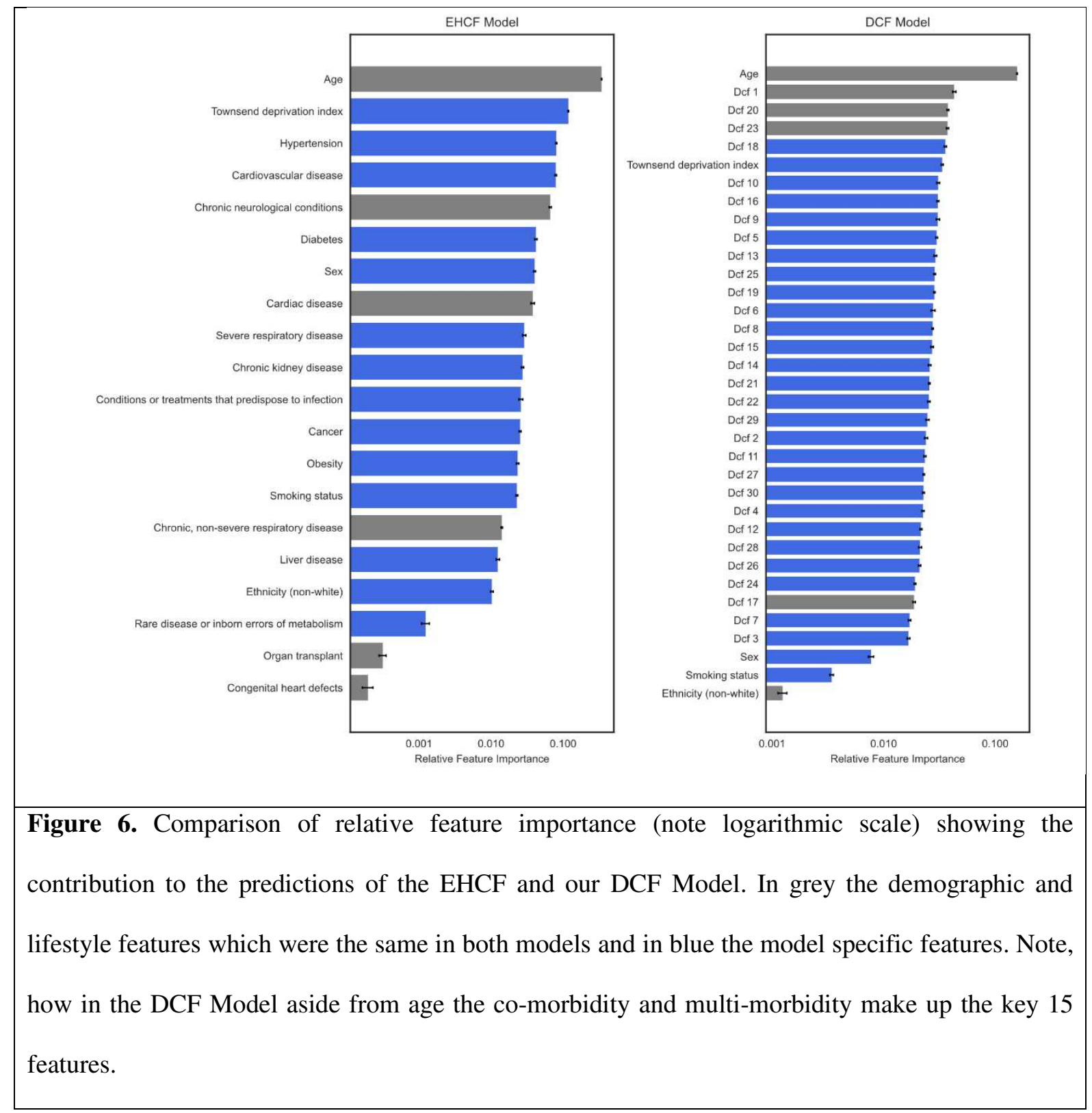




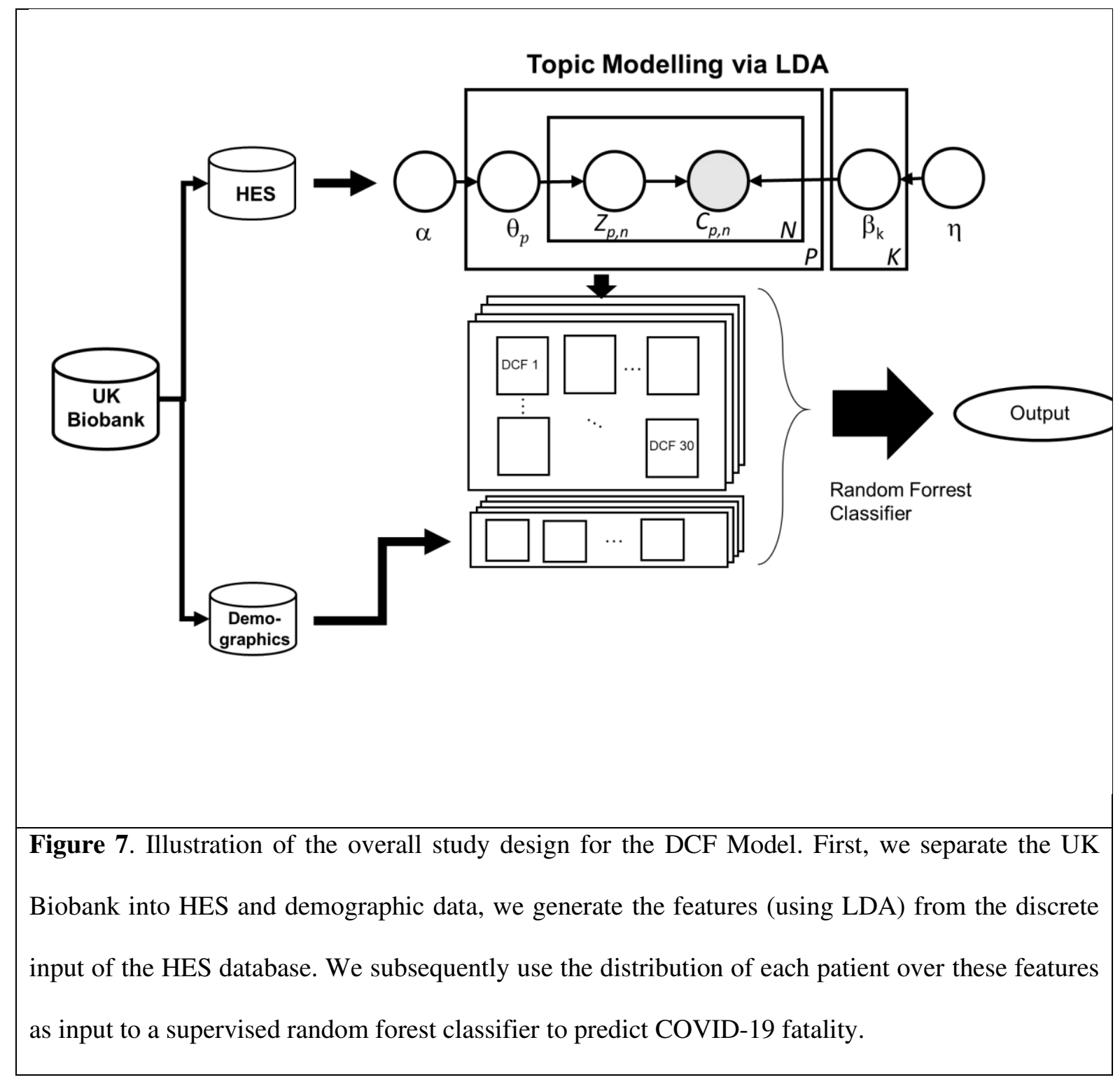

Box 1. Hand-Crafted Predictor variables

\section{Demographics}

- Age in years

- Townsend deprivation index

- Ethnicity 


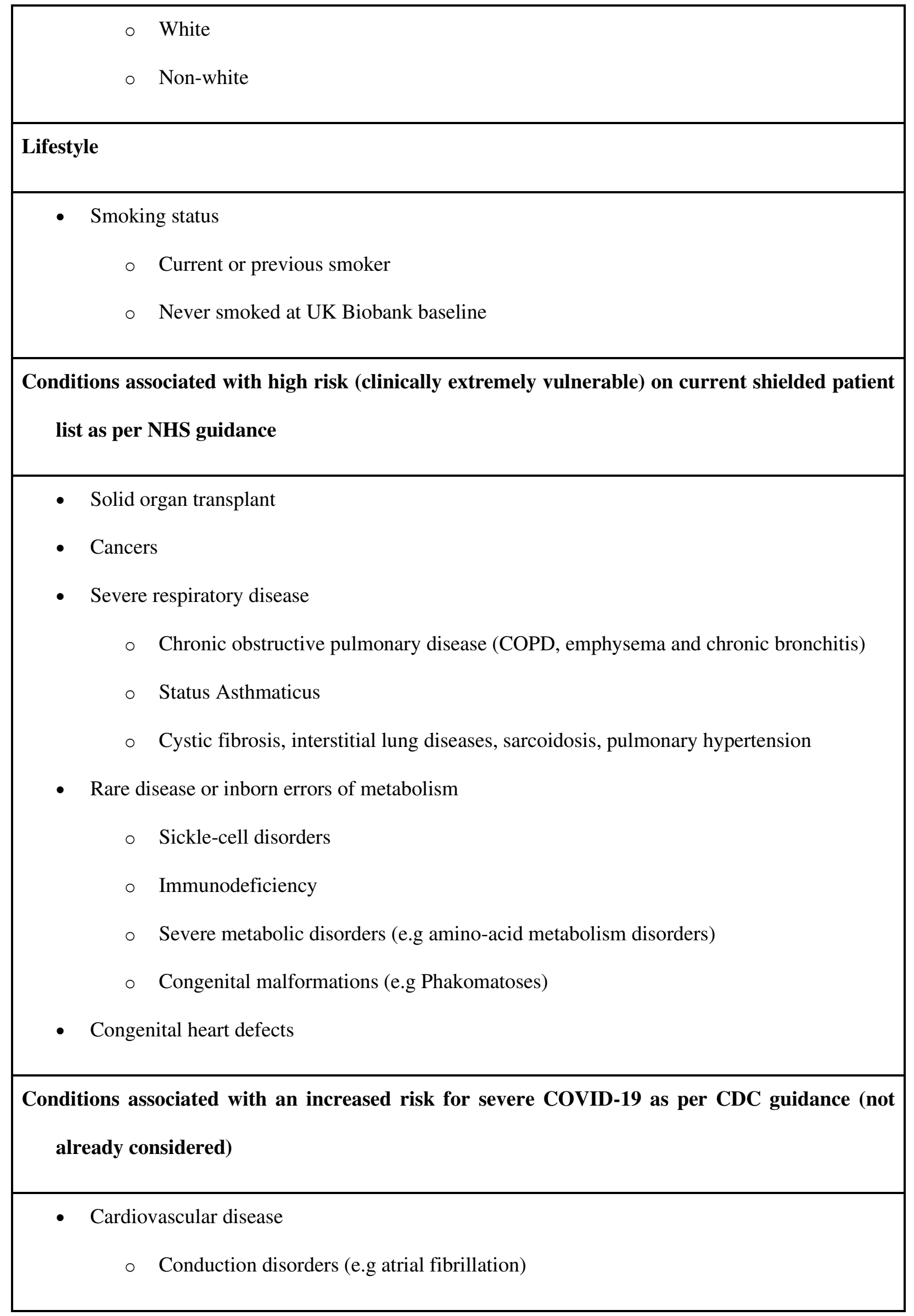


- Past cardiovascular event (e.g myocardial infarction, stroke, angina)

- Peripheral vascular disease

$\circ$ Endocarditis

- HIV

Conditions associated with a moderate risk (clinically vulnerable) as per NHS guidance

- Chronic, non-severe respiratory disease
$\circ$ Asthma
- Hypersensitivity pneumonitis

- Chronic kidney disease

$\circ \quad \mathrm{CKD}>=$ stage 3

- End stage renal failure involving dialysis

- End stage renal failure involving a transplant

- Cardiac disease
- Heart failure
- Valve disorders

- Liver disease
$\circ$ Chronic hepatitis
- Alcoholic liver disease
- Toxic liver disease
- Other causes of liver cirrhosis

- Chronic neurological conditions
- Motor neurone disease
- Parkinson's disease
- Multiple sclerosis 


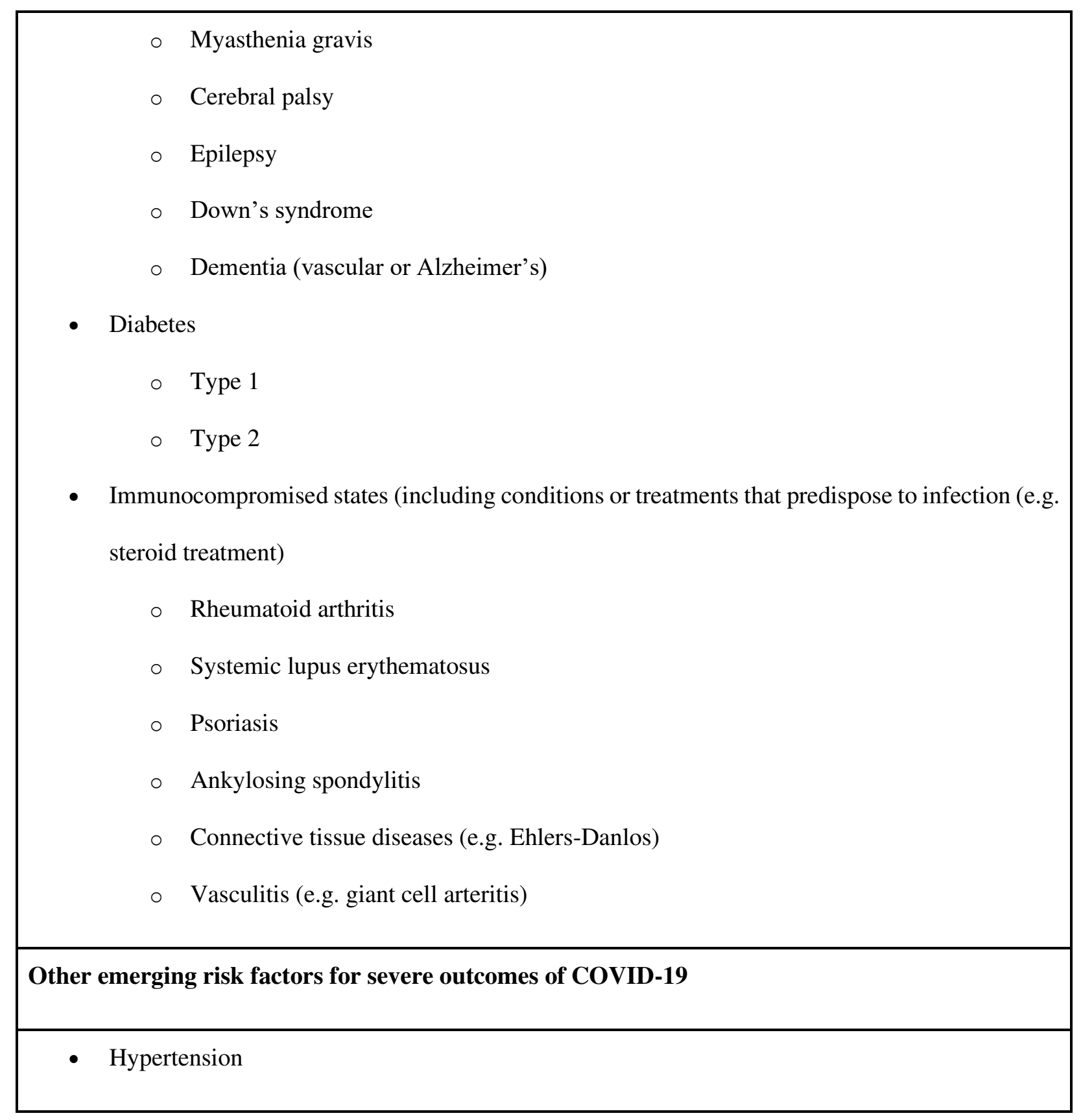

Table 1. Descriptive characteristics of the UK Biobank cohort by COVID-19 fatality and Odds ratios and $95 \%$ confidence intervals for COVID-19-related death

\begin{tabular}{|l|l|l|l|l|}
\hline & $\begin{array}{l}\text { COVID-19- } \\
\text { related death }\end{array}$ & Rest of the & OR $(95 \%$ CI $)$ & Fully adjusted OR \\
$(n=349)$ & $2150)$ & $(\mathrm{ns}=$ & & \\
\hline
\end{tabular}




\begin{tabular}{|c|c|c|c|c|}
\hline Age 50-59 & $14(4.01 \%)$ & $\begin{array}{l}619 \\
(28.79 \%)\end{array}$ & 1 & 1 \\
\hline Age 60-69 & $55(15.76 \%)$ & $\begin{array}{l}537 \\
(24.98 \%)\end{array}$ & $4.53(2.49-8.23)$ & $3.56(1.93-6.54)$ \\
\hline Age 70-79 & $234(67.05 \%)$ & $\begin{array}{l}894 \\
(41.58 \%)\end{array}$ & $11.57(6.68-20.04)$ & $8.00(4.49-14.25)$ \\
\hline Age $80+$ & $46(13.18 \%)$ & $100(4.65 \%)$ & $20.34(10.78-38.36)$ & $14.03(7.18-27.44)$ \\
\hline Sex & $221(63.32 \%)$ & $\begin{array}{l}1063 \\
(49.44 \%)\end{array}$ & $1.77(1.40-2.23)$ & $1.50(1.16-1.94)$ \\
\hline Deprivation Q1 & $64(18.34 \%)$ & $\begin{array}{l}389 \\
(18.09 \%)\end{array}$ & 1 & 1 \\
\hline Deprivation Q2 & $69(19.77 \%)$ & $\begin{array}{l}427 \\
(19.86 \%)\end{array}$ & $0.98(0.68-1.42)$ & $0.96(0.65-1.41)$ \\
\hline Deprivation Q3 & $80(22.92 \%)$ & $\begin{array}{l}567 \\
(26.37 \%)\end{array}$ & $0.86(0.60-1.22)$ & $0.86(0.59-1.25)$ \\
\hline Depreviation Q4 & $136(38.97 \%)$ & $\begin{array}{l}767 \\
(35.67 \%)\end{array}$ & $1.08(0.78-1.49)$ & $1.11(0.78-1.57)$ \\
\hline $\begin{array}{l}\text { Ethnicity (non- } \\
\text { white) }\end{array}$ & $35(10.03 \%)$ & $\begin{array}{l}243 \\
(11.30 \%)\end{array}$ & $0.87(0.60-1.27)$ & $1.40(0.91-2.14)$ \\
\hline Obesity & $67(19.20 \%)$ & $\begin{array}{l}279 \\
(12.98 \%)\end{array}$ & $1.59(1.19-2.14)$ & $1.24(0.89-1.74)$ \\
\hline Smoking status & $240(68.77 \%)$ & $\begin{array}{l}1326 \\
(61.67 \%)\end{array}$ & $1.37(1.07-1.74)$ & $1.10(0.84-1.44)$ \\
\hline Organ transplant & $3(0.86 \%)$ & $19(0.88 \%)$ & $0.97(0.29-3.30)$ & $0.60(0.17-2.17)$ \\
\hline Cancer & $97(27.79 \%)$ & $\begin{array}{l}432 \\
(20.09 \%)\end{array}$ & $1.53(1.18-1.98)$ & $1.13(0.86-1.48)$ \\
\hline
\end{tabular}




\begin{tabular}{|c|c|c|c|c|}
\hline $\begin{array}{l}\text { Severe respiratory } \\
\text { disease }\end{array}$ & $69(19.77 \%)$ & $\begin{array}{l}237 \\
(11.02 \%)\end{array}$ & $1.99(1.48-2.67)$ & $1.03(0.73-1.45)$ \\
\hline $\begin{array}{l}\text { Rare disease or } \\
\text { inborn errors of } \\
\text { metabolism }\end{array}$ & $6(1.72 \%)$ & $18(0.84 \%)$ & $2.07(0.82-5.26)$ & $1.89(0.71-5.04)$ \\
\hline $\begin{array}{l}\text { Congenital heart } \\
\text { defects }\end{array}$ & $4(1.15 \%)$ & $11(0.51 \%)$ & $2.25(0.71-7.12)$ & $1.74(0.49-6.09)$ \\
\hline $\begin{array}{l}\text { Cardiovascular } \\
\text { disease }\end{array}$ & $206(59.03 \%)$ & $\begin{array}{l}761 \\
(35.40 \%)\end{array}$ & $2.63(2.09-3.31)$ & $1.09(0.81-1.46)$ \\
\hline $\begin{array}{l}\text { Chronic, non- } \\
\text { severe respiratory } \\
\text { disease }\end{array}$ & $52(14.90 \%)$ & $\begin{array}{l}302 \\
(14.05 \%)\end{array}$ & $1.07(0.78-1.47)$ & $0.87(0.61-1.24)$ \\
\hline $\begin{array}{l}\text { Chronic kidney } \\
\text { disease }\end{array}$ & $59(16.91 \%)$ & $190(8.84 \%)$ & $2.10(1.53-2.88)$ & $0.97(0.68-1.40)$ \\
\hline Cardiac disease & $71(20.34 \%)$ & $211(9.81 \%)$ & $2.35(1.74-3.16)$ & $1.21(0.86-1.71)$ \\
\hline Liver disease & $42(12.03 \%)$ & $176(8.19 \%)$ & $1.53(1.07-2.19)$ & $1.07(0.73-1.59)$ \\
\hline $\begin{array}{l}\text { Chronic } \\
\text { neurological } \\
\text { conditions }\end{array}$ & $77(22.06 \%)$ & $\begin{array}{l}215 \\
(10.00 \%)\end{array}$ & $2.55(1.91-3.40)$ & $1.64(1.20-2.24)$ \\
\hline Diabetes & $100(28.65 \%)$ & $\begin{array}{l}353 \\
(16.42 \%)\end{array}$ & $2.04(1.58-2.65)$ & $1.21(0.89-1.63)$ \\
\hline $\begin{array}{l}\text { Conditions or } \\
\text { treatments that } \\
\text { predispose to } \\
\text { infection }\end{array}$ & $50(14.33 \%)$ & $157(7.30 \%)$ & $2.12(1.51-2.98)$ & $1.52(1.05-2.20)$ \\
\hline Hypertension & $238(68.19 \%)$ & $\begin{array}{l}942 \\
(43.81 \%)\end{array}$ & $2.75(2.16-3.50)$ & $1.34(1.00-1.81)$ \\
\hline
\end{tabular}


Table 2. Performance of all prediction models under consideration

\begin{tabular}{|c|c|c|}
\hline Predictor Set & \multicolumn{2}{|c|}{ Results } \\
\hline & AUC-ROC & 95\% CI \\
\hline Baseline & 0.709 & $0.692-0.727$ \\
\hline EHCF - Model & 0.734 & $0.704-0.764$ \\
\hline DCF - Model & 0.730 & $0.700-0.760$ \\
\hline
\end{tabular}

\section{Supplementary Material}

Supplementary Box 1. Chapter number and description for each of the 22 chapter's making up the ICD-10 clinical codes

I: Certain infectious and parasitic diseases'
II: Neoplasms
III: Diseases of the blood and blood-forming organs and certain disorders involving the immune
mechanism
IV: Endocrine, nutritional and metabolic diseases
V: Mental and behavioural disorders



$\mathrm{VI}$ : Diseases of the nervous system
VII: Diseases of the eye and adnexia
VIII: Diseases of the ear and mastoid process
IX: Diseases of the circulatory system
$\mathrm{X}$ : Diseases of the respiratory system
$\mathrm{XI}$ : K00-K95', 'Diseases of the digestive system'
XII: Diseases of the skin and subcutaneous tissue
XIII: Diseases of the musculoskeletal system and connective tissue
XIV: Diseases of the genitourinary system
$\mathrm{XV}$ : Pregnancy, childbirth and the puerperium
$\mathrm{XVI}$ : Certain conditions originating in the perinatal period

XVII: Congenital malformations, deformations and chromosomal abnormalities

XVIII: Symptoms, signs and abnormal clinical and laboratory findings, not elsewhere classified

XIX: Injury, poisoning and certain other consequences of external causes

XX: External causes of morbidity and mortality

XXI: Factors influencing health status and contact with health services

XXII: Codes for special purposes

Supplementary Box 2. Top 15 disease codes (in rank order) from all 30 topics of the 30-topic LDA model used in the DCF Model

\section{DCF 1:}

Rheumatoid arthritis

Chemotherapy session for neoplasm

Rheumatoid arthritis, unspecified (Site unspecified)

Chronic lymphocytic leukaemia

Diffuse non-Hodgkin's lymphoma - Large cell (diffuse)

Personal history of malignant neoplasms of lymphoid, haematopoietic and related tissues

Non-Hodgkin's lymphoma, unspecified type

Rheumatoid arthritis, unspecified (Multiple sites)

Polycythaemia vera

Personal history of chemotherapy for neoplastic disease

Follicular non-Hodgkin's lymphoma, unspecified 


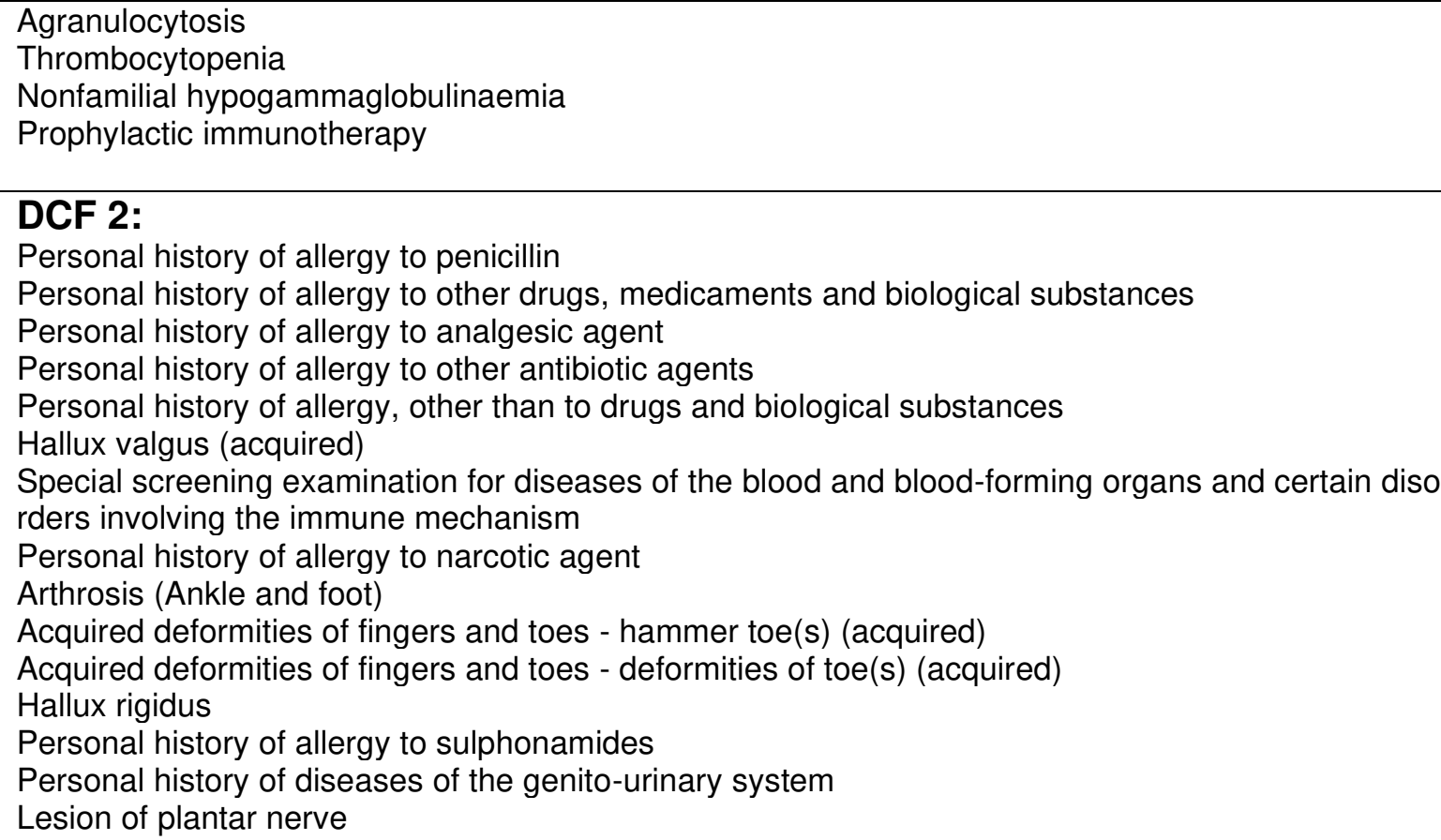

DCF 2:

Personal history of allergy to penicillin

Personal history of allergy to other drugs, medicaments and biological substances

Personal history of allergy to analgesic agent

Personal history of allergy to other antibiotic agents

Personal history of allergy, other than to drugs and biological substances

Hallux valgus (acquired)

Special screening examination for diseases of the blood and blood-forming organs and certain diso rders involving the immune mechanism

Personal history of allergy to narcotic agent

Arthrosis (Ankle and foot)

Acquired deformities of fingers and toes - hammer toe(s) (acquired)

Acquired deformities of fingers and toes - deformities of toe(s) (acquired)

Hallux rigidus

Personal history of allergy to sulphonamides

Personal history of diseases of the genito-urinary system

Lesion of plantar nerve

\section{DCF 3:}

Disorders of iron metabolism

Multiple myeloma

Bronchiectasis

Acute myeloid leukaemia

Transplanted organ and tissue status

Personal history of infectious and parasitic diseases

Personal history of chemotherapy for neoplastic disease

Acquired absence of lung [part of]

Personal history of malignant neoplasm of trachea, bronchus and lung

Malignant neoplasm of bronchus and lung

Malignant neoplasm of bronchus and lung - upper lobe, bronchus or lung

Unspecified acute lower respiratory infection

Abnormal findings on diagnostic imaging of lung

Intrathoracic lymph nodes

Personal history of diseases of the respiratory system

\section{DCF 4:}

Diaphragmatic hernia without obstruction or gangrene

Gastro-oesophageal reflux disease without oesophagitis

Gastritis, unspecified

Gastro-oesophageal reflux disease with oesophagitis

Iron deficiency anaemia, unspecified

Oesophagitis

Barrett's oesophagus

Anaemia, unspecified

Personal history of diseases of the digestive system

Dyspepsia

Polyp of stomach and duodenum

Duodenitis

Ulcer of oesophagus

Other gastritis

Dysphagia

\section{DCF 5:}

Personal history of psychoactive substance abuse 


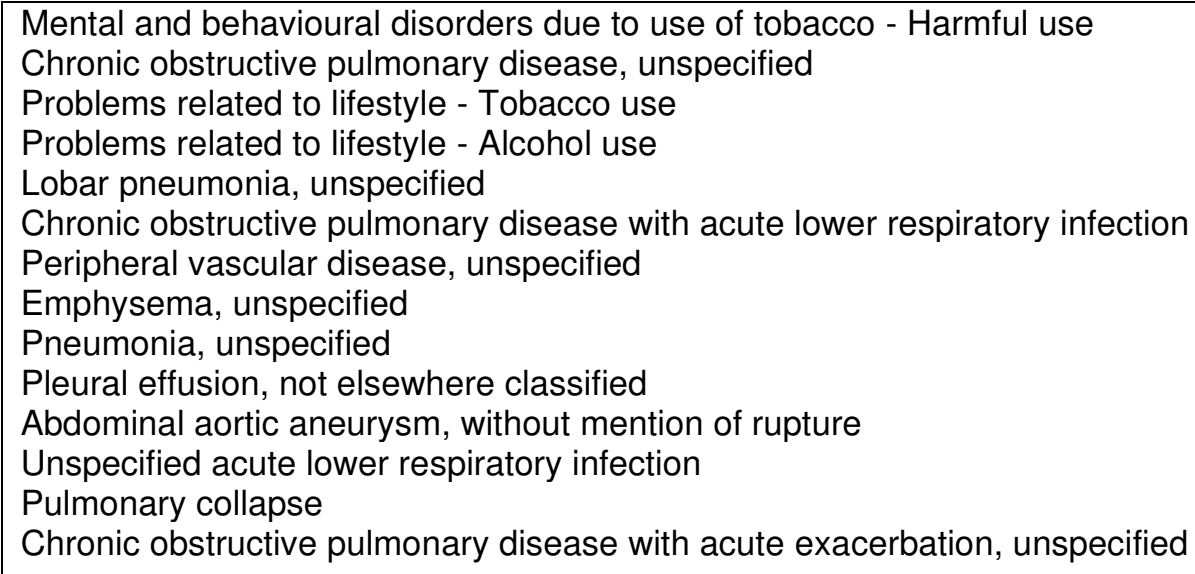

\section{DCF 6:}

Malignant neoplasm of prostate

Acquired absence of genital organ(s)

Personal history of malignant neoplasm of genital organs

Personal history of irradiation

Malignant neoplasm of ovary

Malignant neoplasm of corpus uteri - endometrium

Family history of malignant neoplasm of genital organs

Other specified abnormal findings of blood chemistry

Fitting and adjustment of urinary device

Personal history of chemotherapy for neoplastic disease

Intrapelvic lymph nodes

Myelodysplastic syndrome, unspecified

Secondary malignant neoplasm of bone and bone marrow

Naevus, nonneoplastic

Other hypertrophic disorders of skin

\section{DCF 7:}

Chest pain, unspecified

Radiotherapy session

Calculus of ureter

Internal haemorrhoids with other complications

Benign lipomatous neoplasm of skin and subcutaneous tissue of trunk

Dyspnoea

Viral infection, unspecified

Unspecified renal colic

Benign lipomatous neoplasm of skin and subcutaneous tissue of limbs

Observation for suspected myocardial infarction

Lymphoedema, not elsewhere classified

Bilateral inguinal hernia, without obstruction or gangrene

R07 Pain in throat and chest - R07.2 Precordial pain

R04 Haemorrhage from respiratory passages - R04.0 Epistaxis

Cardiac murmur, unspecified

\section{DCF 8:}

Personal history of long-term (current) use of anticoagulants

Atrial fibrillation and flutter

Personal history of diseases of the circulatory system

Atrial fibrillation and atrial flutter, unspecified

Presence of cardiac pacemaker

Congestive heart failure

Paroxysmal atrial fibrillation

Pulmonary embolism without mention of acute cor pulmonale

Aortic (valve) stenosis 


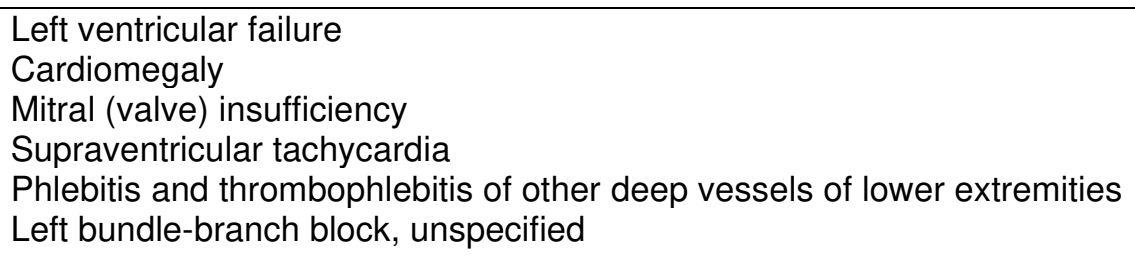

\section{DCF 9:}

Malignant neoplasm of breast

Chemotherapy session for neoplasm

Personal history of malignant neoplasm of breast

Axillary and upper limb lymph nodes

Acquired absence of breast(s)

Malignant neoplasm of breast - upper-outer quadrant of breast

Secondary malignant neoplasm of bone and bone marrow

Family history of malignant neoplasm of breast

Personal history of irradiation

Personal history of chemotherapy for neoplastic disease

Follow-up care involving plastic surgery of breast

Lymph nodes of head, face and neck

Malignant neoplasm of breast - upper-inner quadrant of breast

Secondary malignant neoplasm of lung

Carcinoma in situ of breast - intraductal carcinoma in situ

\section{DCF 10:}

Presence of orthopaedic joint implants

Gonarthrosis, unspecified

Coxarthrosis, unspecified

Arthrosis, unspecified

Obesity, unspecified

Polyarthrosis, unspecified

Surgical operation with implant of artificial internal device

Arthritis, unspecified

Other primary gonarthrosis

Arthrosis, unspecified (Site unspecified)

Derangement of meniscus due to old tear or injury

Carpal tunnel syndrome

Other primary coxarthrosis

Mechanical complication of internal joint prosthesis

Derangement of meniscus due to old tear or injury

\section{DCF 11:}

Hypothyroidism, unspecified

Multiple sclerosis

Dental caries, unspecified

Postprocedural hypothyroidism

Thyrotoxicosis, unspecified

Retained dental root

Special screening examination for other specified diseases and disorders

Hypopituitarism

Thyrotoxicosis with diffuse goitre

Vitamin B12 deficiency anaemia due to intrinsic factor deficiency

Benign neoplasm of other and unspecified endocrine glands - pituitary gland

Benign neoplasm of breast

Malignant neoplasm of thyroid gland

Primary adrenocortical insufficiency

Other benign neoplasms of skin - skin of eyelid, including canthus

DCF 12: 


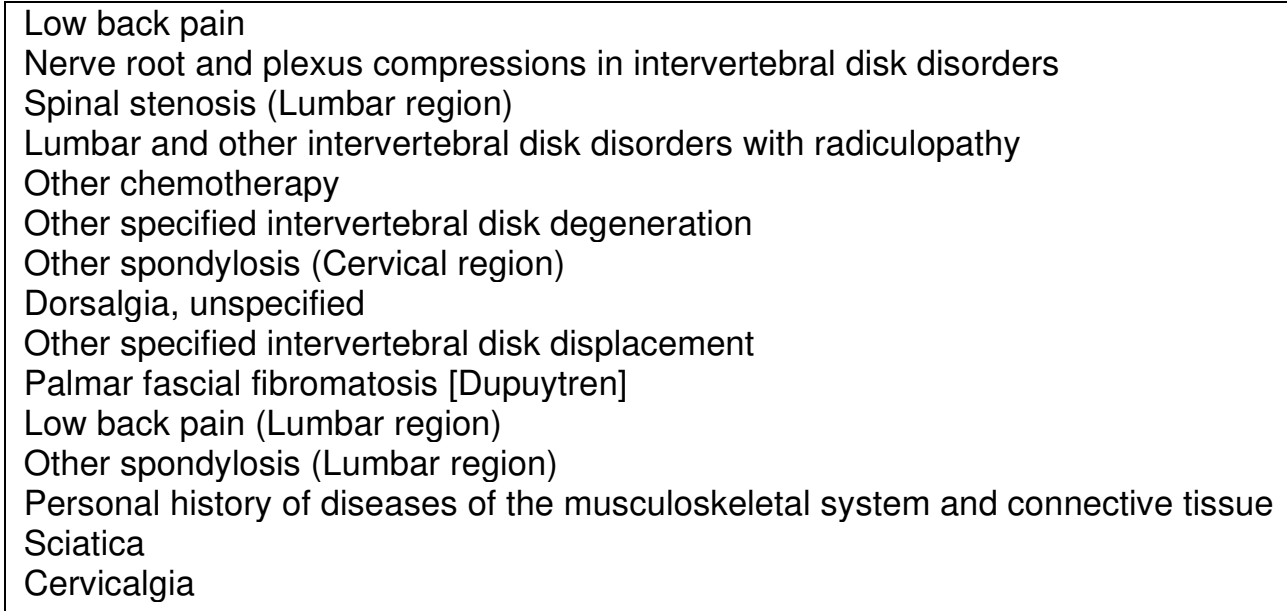

\section{DCF 13:}

Personal history of malignant neoplasm of urinary tract

Excessive and frequent menstruation with regular cycle

Leiomyoma of uterus, unspecified

Postmenopausal bleeding

Malignant neoplasm of bladder

Calculus of kidney

Polyp of corpus uteri

Personal history of diseases of the genito-urinary system

Follow-up examination after surgery for malignant neoplasm

Other and unspecified ovarian cysts

Acquired absence of kidney

Polyp of cervix uteri

Incomplete uterovaginal prolapse

pelvic peritoneal adhesions

Excessive and frequent menstruation with irregular cycle

\section{DCF 14:}

Other chemotherapy

Raynaud's syndrome

Other interstitial pulmonary diseases with fibrosis

Sicca syndrome [Sjogren]

Polyneuropathy, unspecified

Systemic lupus erythematosus, unspecified

Interstitial cystitis (chronic)

Melanocytic naevi of other and unspecified parts of face

Sarcoidosis, unspecified

Ankylosing spondylitis

Other inflammatory polyneuropathies

Other disorders of arteries

Other specified polyneuropathies

Other chronic pain

Wegener's granulomatosis

\section{DCF 15:}

Other and unspecified abdominal pain

Nausea and vomiting

Headache

Non-infective gastro-enteritis and colitis, unspecified

Change in bowel habit

Constipation

Other chest pain

Pain localised to other parts of lower abdomen 


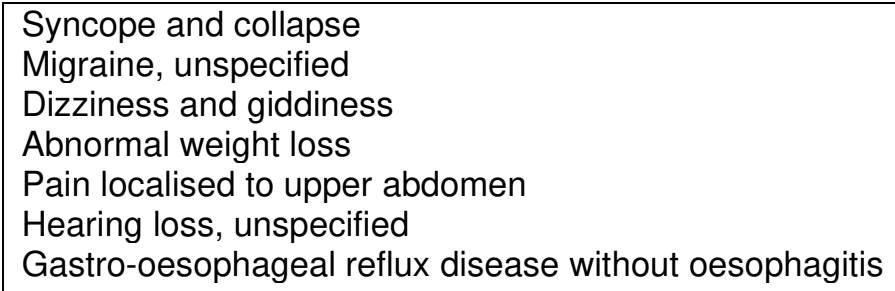

\section{DCF 16:}

Hyperplasia of prostate

Unilateral or unspecified inguinal hernia, without obstruction or gangrene

Personal history of malignant neoplasms of other organs and systems

Unspecified haematuria

Other malignant neoplasms of skin and unspecified parts of face

Other specified disorders of bladder

Retention of urine

Urethral stricture, unspecified

Personal history of diseases of the genito-urinary system

Disorder of skin and subcutaneous tissue, unspecified

Trichilemmal cyst

Polyuria

Urinary tract infection, site not specified

Seborrhoeic keratosis

Other and unspecified symptoms and signs involving the urinary system

\section{DCF 17:}

Personal history of malignant neoplasm of digestive organs

Acquired absence of other parts of digestive tract

Chemotherapy session for neoplasm

Malignant neoplasm of rectum

Intestinal bypass and anastomosis status

Intra-abdominal lymph nodes

Malignant neoplasm of colon - sigmoid colon

lleostomy status

Secondary malignant neoplasm of liver

0 Follow-up examination after surgery for malignant neoplasm

5 Arthropathic psoriasis

Personal history of chemotherapy for neoplastic disease

Colostomy status

Malignant neoplasm of colon - colon

Malignant neoplasm of colon - caecum

\section{DCF 18:}

Personal history of diseases of the circulatory system

Urinary tract infection, site not specified

Personal history of diseases of the nervous system and sense organs

Acute renal failure, unspecified

Tendency to fall, not elsewhere classified

Physical therapy

Parkinson's disease

Hypo-osmolality and hyponatraemia

Other and unspecified abnormalities of gait and mobility

Constipation

Occupational therapy and vocational rehabilitation, not elsewhere classified

Escherichia coli [E. coli] as the cause of diseases

Hemiplegia, unspecified

Volume depletion

Disorientation, unspecified 


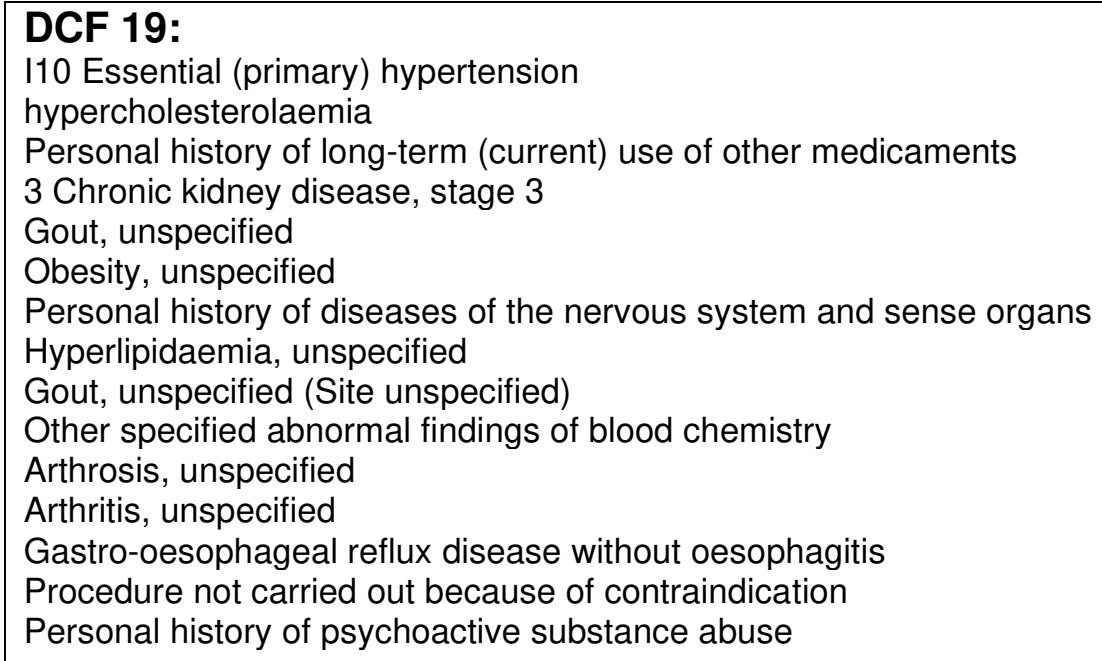

\section{DCF 20:}

Diverticular disease of large intestine without perforation or abscess

Polyp of colon

Diverticular disease of intestine, part unspecified, without perforation or abscess

Family history of malignant neoplasm of digestive organs

Unspecified haemorrhoids without complication

Personal history of diseases of the digestive system

Special screening examination for neoplasm of intestinal tract

Haemorrhage of anus and rectum

Personal history of other neoplasms

Rectal polyp

Sigmoid colon

Gastro-intestinal haemorrhage, unspecified

Follow-up examination after surgery for other conditions

Haemorrhoids, unspecified

Change in bowel habit

\section{DCF 21:}

Cataract, unspecified

Senile nuclear cataract

Presence of intraocular lens

Glaucoma

Degeneration of macula and posterior pole

Psoriasis, unspecified

Sterilisation

Senile cataract

Senile incipient cataract

6 Personal history of diseases of the nervous system and sense organs

Primary open-angle glaucoma

Myopia

Family history of eye and ear disorders

After-cataract

Arthritis, unspecified

\section{DCF 22:}

Depressive episode

Anxiety disorder

Mental and behavioural disorders due to use of alcohol - harmful use

Cellulitis of other parts of limb

Mental and behavioural disorders due to use of alcohol - dependence syndrome

Bipolar affective disorder, unspecified

Mixed anxiety and depressive disorder 


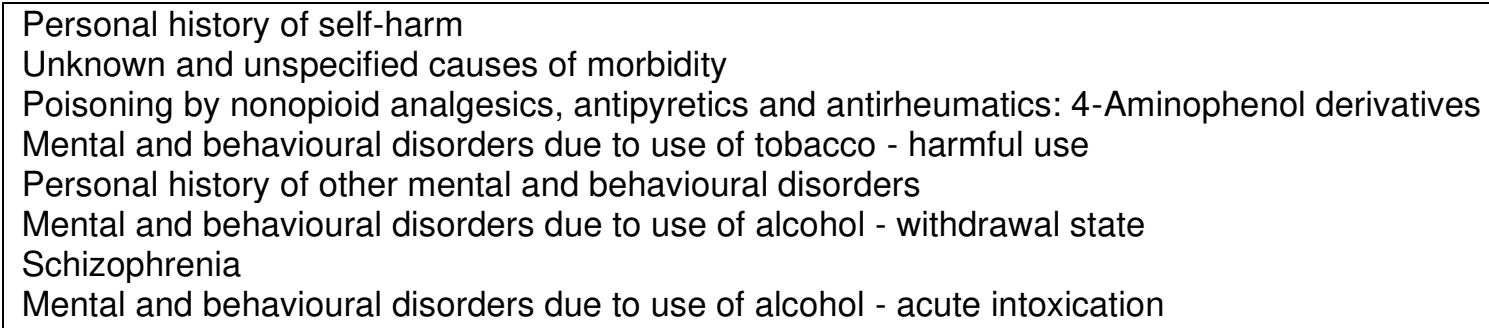

\section{DCF 23:}

Non-insulin-dependent diabetes mellitus apnoea

\section{DCF 24:}

Extracorporeal dialysis

Chronic kidney disease, stage 5

Chronic renal failure, unspecified

End-stage renal disease

Polymyalgia rheumatica

Kidney transplant status

Dependence on renal dialysis

Hypertensive renal disease with renal failure

Other and unspecified cirrhosis of liver

Chronic kidney disease, stage 4

Portal hypertension

Alcoholic cirrhosis of liver

Unspecified renal failure

Oesophageal varices without bleeding

Ascites

\section{DCF 25:}

Angina pectoris

Atherosclerotic heart disease

Chronic IHD

Old myocardial infarction

Presence of coronary angioplasty implant and graft

Personal history of long-term use of other medicaments

Pure hypercholesterolaemia

Presence of aortocoronary bypass graft

Family history of IHD

Other forms of chronic ischaemic heart disease

Personal history of psychoactive substance abuse

Chest pain

Essential (primary) hypertension

Unstable angina

Hyperlipidaemia, unspecified 


\section{DCF 26:}

Osteoporosis, unspecified

Epilepsy, unspecified

Irritable bowel syndrome without diarrhoea

Crohn's disease, unspecified

Osteoporosis, unspecified (Site unspecified)

Coeliac disease

Fracture of lower end of radius (closed)

Vitamin $\mathrm{D}$ deficiency, unspecified

Other specified disorders of bone density and structure

Arthrosis, unspecified

Deficiency of other specified B group vitamins

Fracture of neck of femur (closed)

Crohn's disease of large intestine

Crohn's disease of small intestine

Primary hyperparathyroidism

\section{DCF 27:}

Carpal tunnel syndrome

Fibromyalgia

Exposure to unspecified factor - unspecified place

Follow-up care involving removal of fracture plate and other internal fixation device

Unspecified fall

Fractures of other parts of lower leg (closed)

wound of finger(s) without damage to nail

Unspecified injury of head

Fall on and from stairs and steps at home

Fall on same level from slipping, tripping and stumbling at home

Fracture of upper end of humerus (closed)

Postviral fatigue syndrome

Open wound of other parts of head

Fall on same level from slipping, tripping and stumbling, unspecified place

Multiple fractures of ribs (closed)

\section{DCF 28:}

Personal history of diseases of the digestive system

Calculus of gallbladder without cholecystitis

Acquired absence of other parts of digestive tract

Calculus of gallbladder with other cholecystitis

following a procedure, not elsewhere classified

Removal of other organ (partial) (total)

Pain localised to upper abdomen

Abnormal results of liver function studies

Other surgical procedures

Peritoneal adhesions

Fatty (change of) liver, not elsewhere classified

Unspecified abdominal pain

Haemorrhage and haematoma complicating a procedure, not elsewhere classified

Calculus of bile duct without cholangitis or cholecystitis

Ventral hernia without obstruction or gangrene

\section{DCF 29:}

Single live birth

Varicose veins of lower extremities without ulcer or inflammation

Ulcerative colitis, unspecified

degree perineal laceration during delivery

First degree perineal laceration during delivery 
Other disorders of synovium and tendon: ganglion

Other specified pregnancy-related conditions

Spontaneous vertex delivery

Labour and delivery complicated by foetal heart rate anomaly

Missed abortion

Maternal care for other specified foetal problems

Prolonged second stage (of labour)

Maternal care due to uterine scar from previous surgery

Prolonged pregnancy

Other and unspecified abdominal pain

DCF 30:

Asthma, unspecified

Unknown and unspecified causes of morbidity

Stress incontinence

Female genital prolapse: cystocele

Impingement syndrome of shoulder

Female genital prolapse: rectocele

Rotator cuff syndrome

Dermatitis, unspecified

Arthrosis, unspecified (Shoulder region)

Unspecified acute lower respiratory infection

Predominantly allergic asthma

Adhesive capsulitis of shoulder

Obesity

Gastro-oesophageal reflux disease without oesophagitis

Other shoulder lesions 
Figures

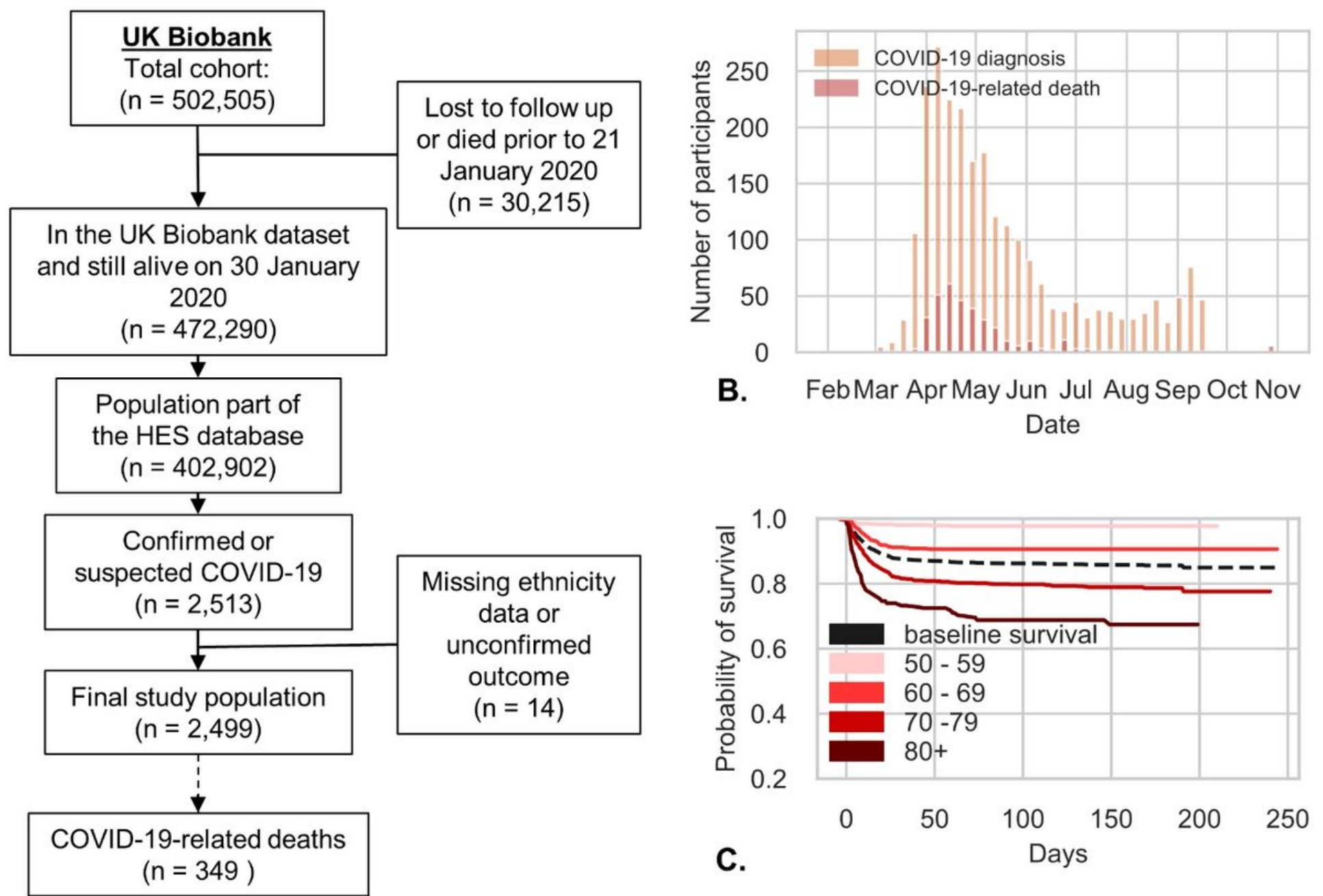

A.

Figure 1

Cohort description. (A) Flowchart diagram of the study cohort. (B) Distribution of COVID-19 diagnosis and death. (C) Kaplan-Meier plots for COVID-19-related death per age group 


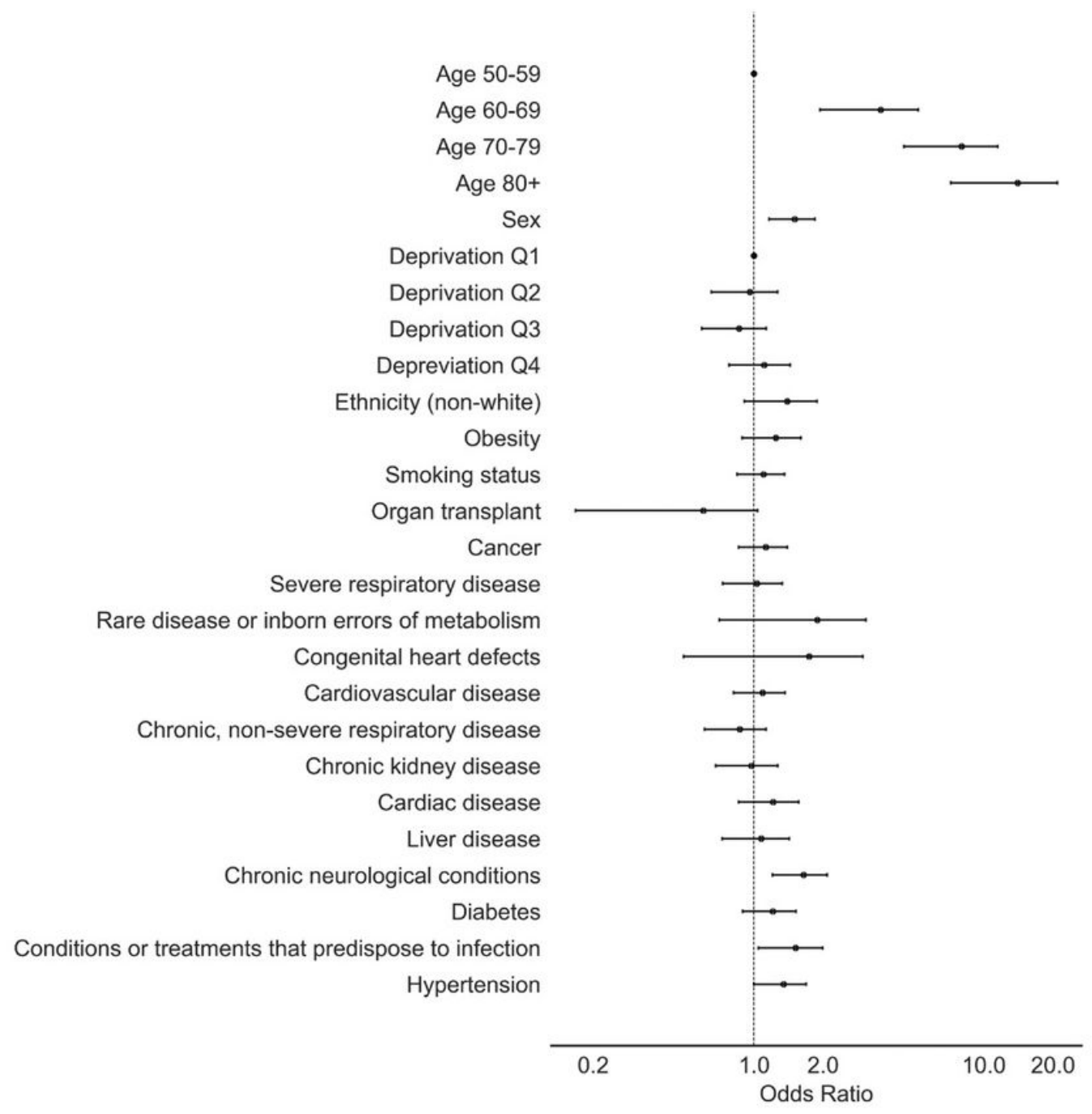

Figure 2

Estimated Odds Ratio for each patient characteristic from a multivariable logistic regression 


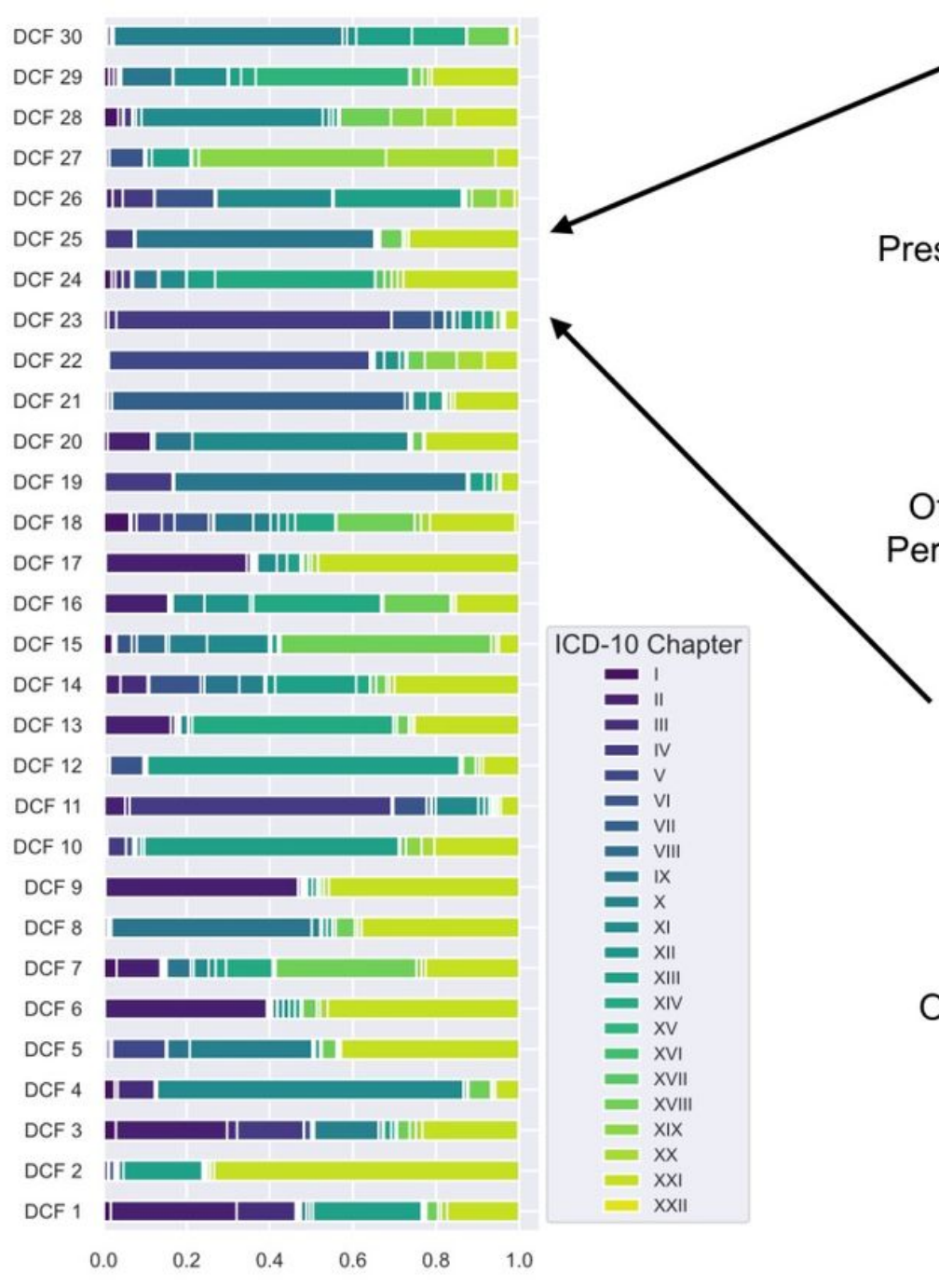

DCF 24: "Cardiovascular"

Angina pectoris

Atherosclerotic heart disease

Chronic IHD

Old myocardial infarction

Presence of coronary angioplasty implant and graft

Personal history of long-term use of other

medicaments

Pure hypercholesterolaemia

Presence of aortocoronary bypass graft

Family history of IHD

Other forms of chronic ischaemic heart disease

Personal history of psychoactive substance abuse

Chest pain

Essential (primary) hypertension

DCF 22: "Diabetes"

Non-insulin-dependent diabetes mellitus

Sleep apnoea

Insulin-dependent diabetes mellitus

Obesity

Diabetic retinopathy

Unspecified diabetes mellitus

Ophthalmic complications of diabetes mellitus

Ulcer of lower limb

Other obesity

Cellulitis of other parts of limb

Acute renal failure

Hypoglycaemia

Chronic renal failure

Figure 3

Topic description. We fit a 30-topic LDA model to 420,000 patients from the UK Biobank. On the left is the distribution of the 22 ICD-10 'Chapters' (Supplementary Box 1) over the 30 topics. On the right are the top 13 ICD-10 codes from topics 22 and 24 (in rank order). 


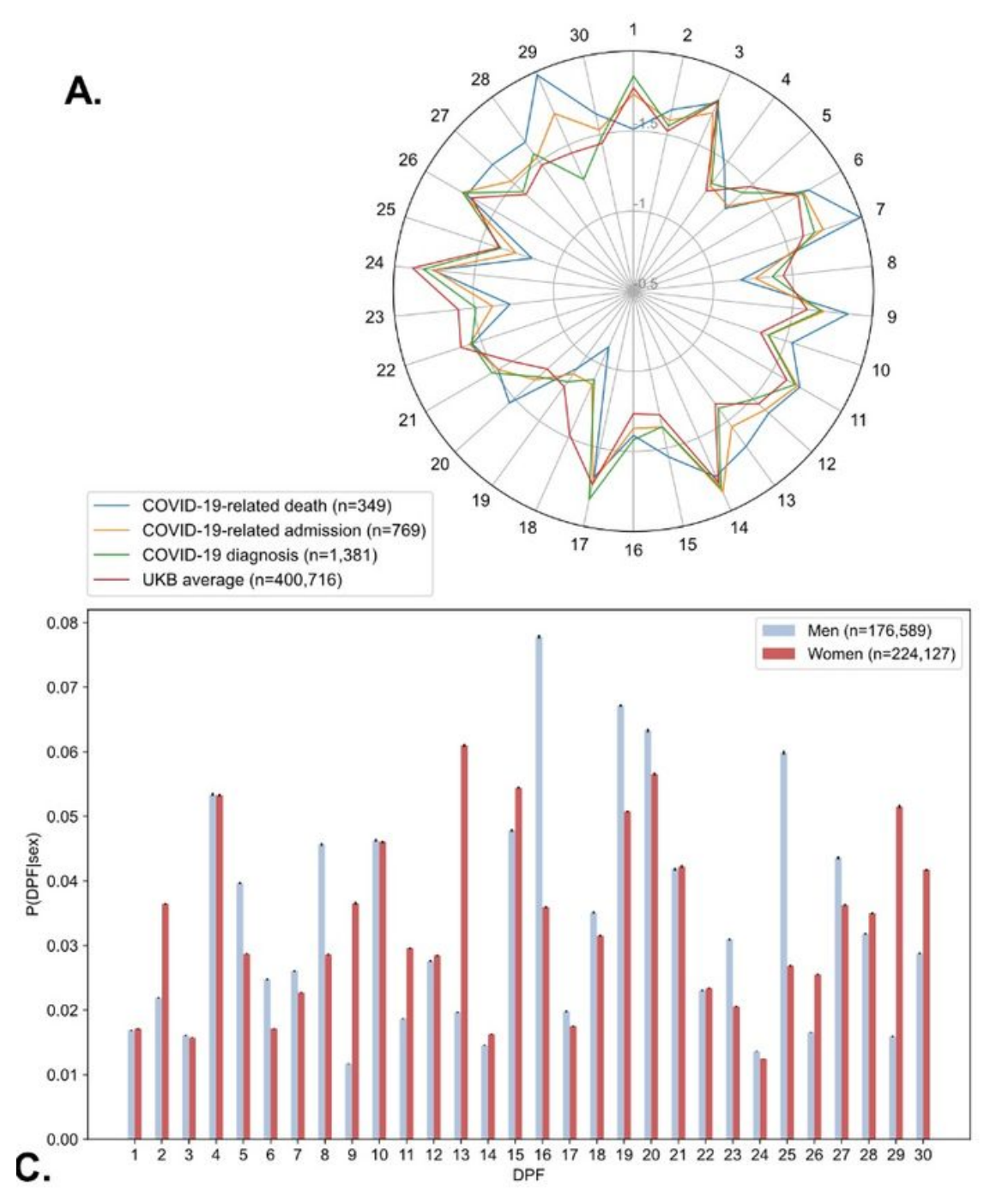

B.
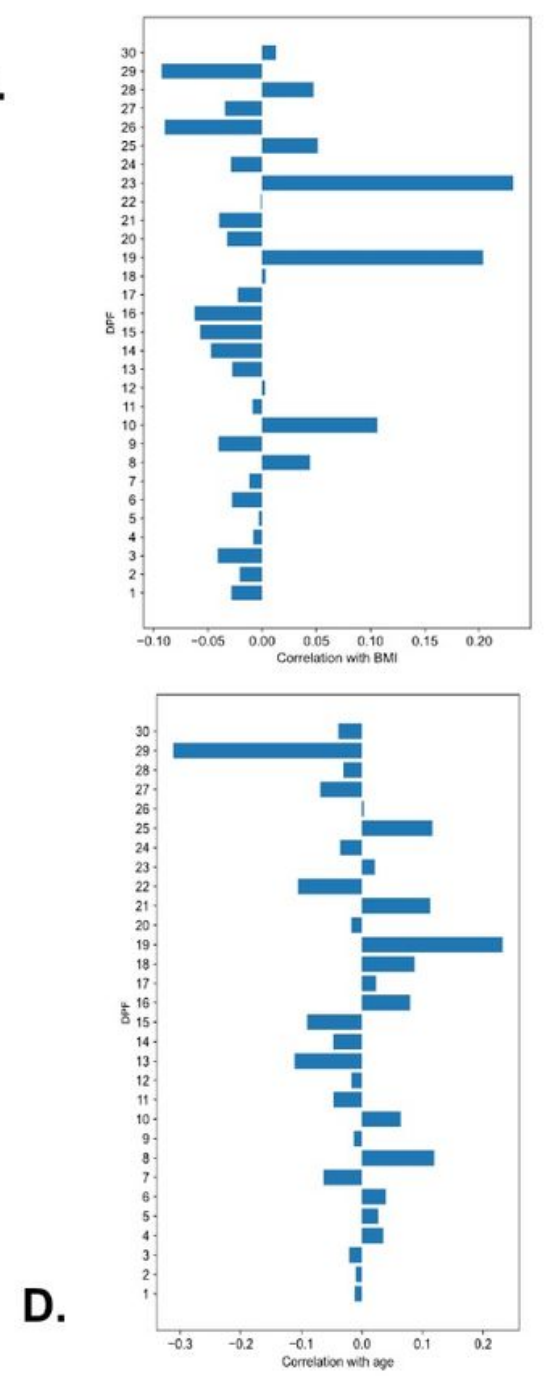

Figure 4

Summary of the Digital Precondition Fingerprints. (A) Details the average probability of belonging to each DPF (log 10 scale) by COVID-19 outcome. (B) Shows the Pearson correlation between each of our DCFs and the patient's BMI. (C) Shows the average probability of belonging to each DCF by gender and (D) shows the Pearson correlation between the each DCF and the patient's age. 


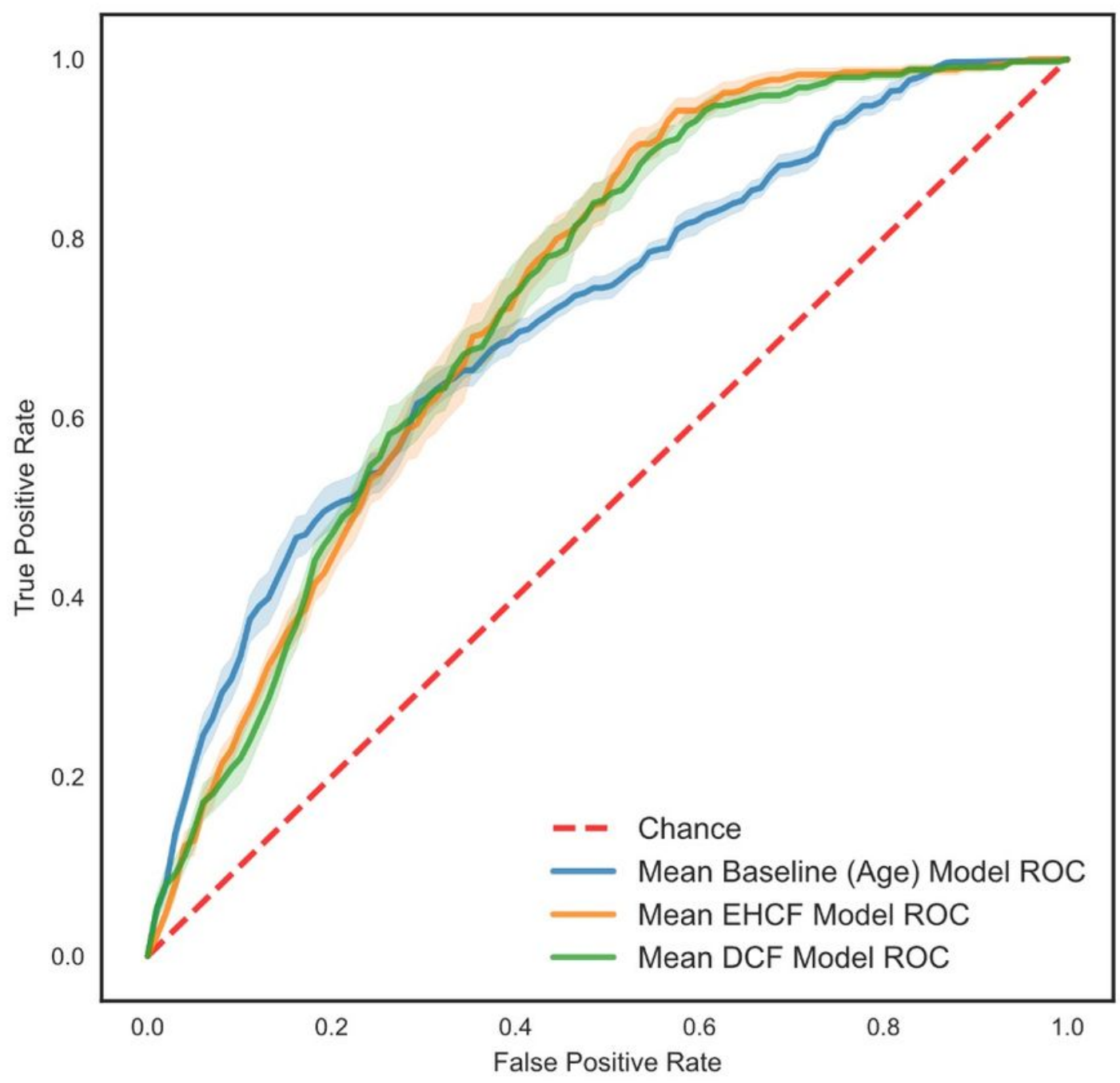

Figure 5

Mean receiver operating characteristic curves derived from predicting COVID-19-related death in the 10Fold Cross Validation (standard error of the mean) for all models. 

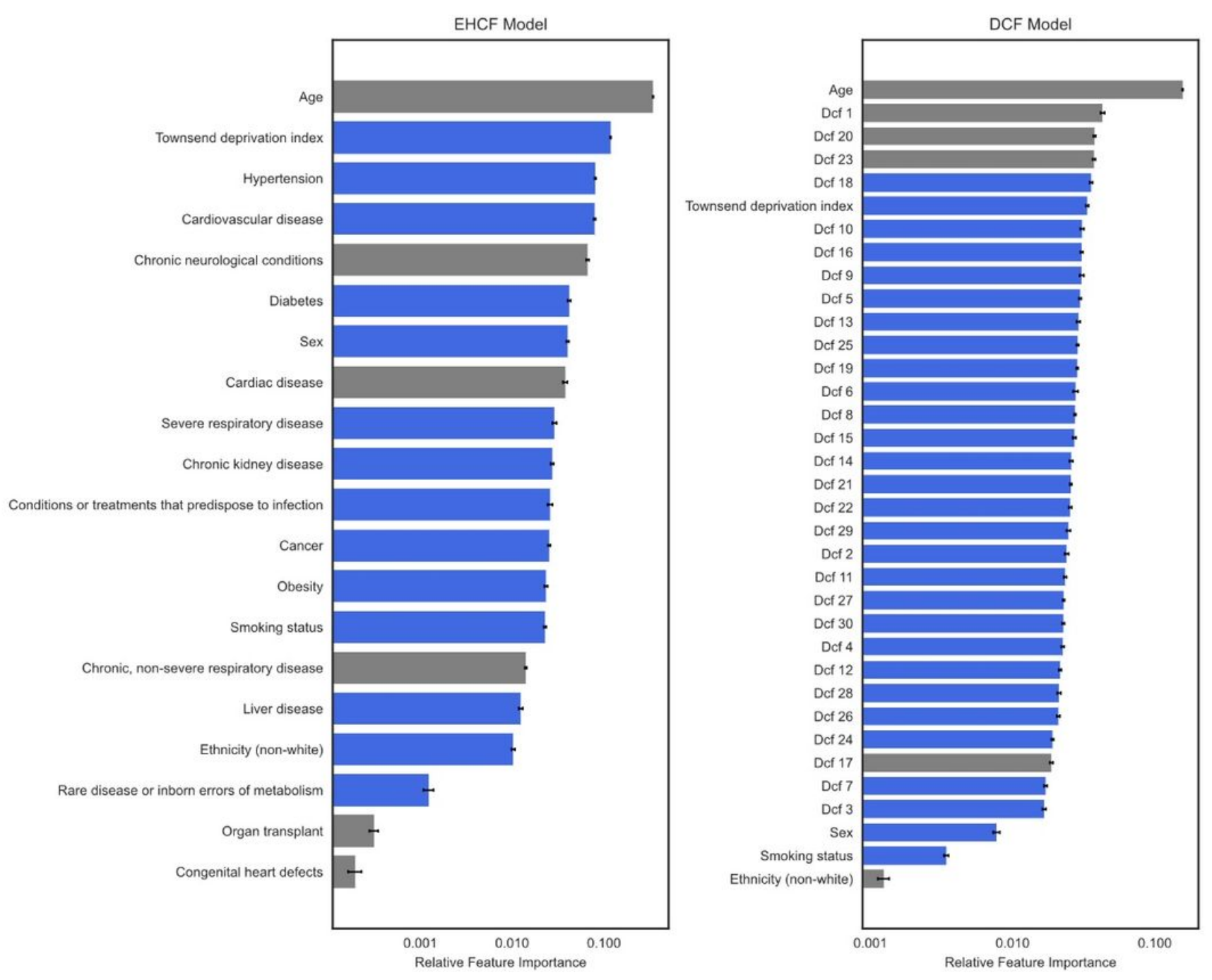

Figure 6

Comparison of relative feature importance (note logarithmic scale) showing the contribution to the predictions of the EHCF and our DCF Model. In grey the demographic and lifestyle features which were the same in both models and in blue the model specific features. Note, how in the DCF Model aside from age the co-morbidity and multi-morbidity make up the key 15 features. 


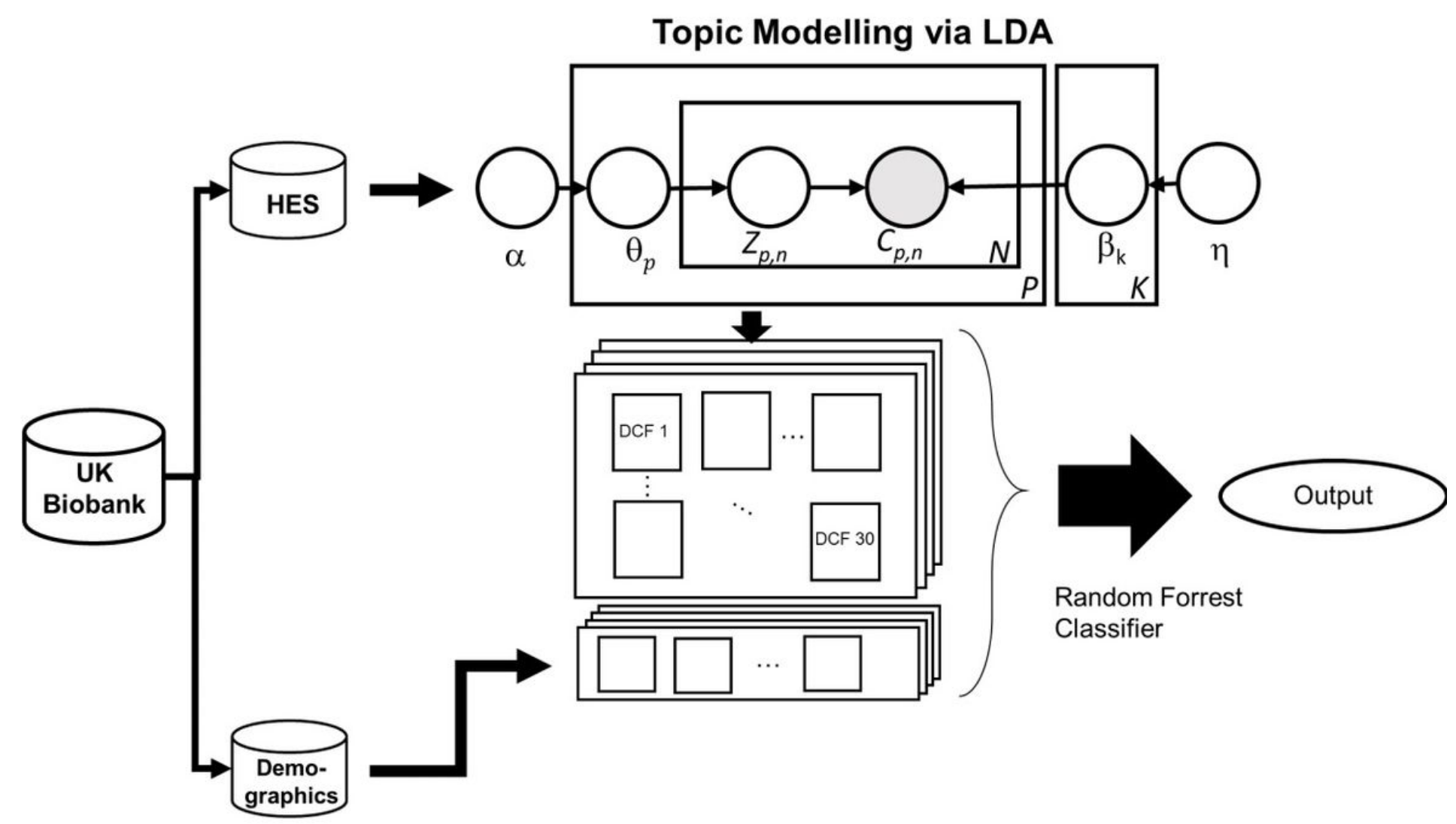

Figure 7

Illustration of the overall study design for the DCF Model. First, we separate the UK Biobank into HES and demographic data, we generate the features (using LDA) from the discrete input of the HES database. We subsequently use the distribution of each patient over these features as input to a supervised random forest classifier to predict COVID-19 fatality. 